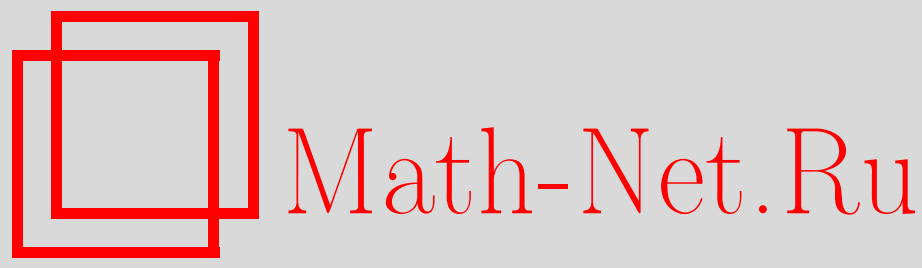

В. Г. Журавлев, Периодические ядерные разложения кубических иррациональностей в цепные дроби, Совр. пробл. матем., 2016, выпуск 23, 43-68

DOI: https://doi.org/10.4213/spm61

Использование Общероссийского математического портала Math-Net.Ru подразумевает, что вы прочитали и согласны с пользовательским соглашением http://www . mathnet.ru/rus/agreement

Параметры загрузки:

IP: 54.196 .121 .252

26 апреля 2023 г., 18:12:06

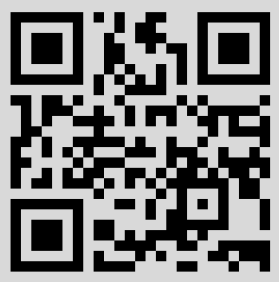




\title{
Периодические ядерные разложения кубических иррациональностей в цепные дроби
}

\author{
В. Г. Журавлёв \\ Владимирский государственный университет \\ vzhuravlev@mail.ru
}

\section{Введение}

0.1. Теорема Лагранжа. Пусть $x=\left(x_{1}, x_{2}\right)$ - произвольная точка с координатами из $\mathbb{R}$ и $F_{x}=\mathbb{Q}\left(x_{1}, x_{2}\right)$ - расширение поля рациональных чисел $\mathbb{Q}$, получающееся добавлением к нему чисел $x_{1}, x_{2}$. Определим степень точки $x$ над $\mathbb{Q}$, полагая $\operatorname{deg}(x)=\operatorname{deg} F_{x}$, где $\operatorname{deg} F_{x}=$ $\left[F_{x}: \mathbb{Q}\right]$ - степень поля $F_{x}$ над $\mathbb{Q}$.

В [1] была доказана

Теорема 0.1. Пусть точка $x=\left(x_{1}, x_{2}\right)$ будет иррачиональной, т.е. числа $1, x_{1}, x_{2}$ линейно независимы над кольцом челых рачиональных чисел $\mathbb{Z}$. Если она при этом является неподвижной точкой некоторого отображения $\delta$ из полугруппы двумерных возвратных отображений $\mathcal{D}$, то ее степень $\operatorname{deg}(x)=3$.

Отображения из $\mathcal{D}$ представляют собой обобщение одномерного возвратного отображения $\delta:[0,1) \rightarrow[0,1)$, определяемого условиями

$$
\delta(x)=\frac{x}{1-x} \quad \text { или } \quad \delta(x)=\frac{2 x-1}{x}
$$

для $0 \leqslant x<1 / 2$ или $1 / 2 \leqslant x<1$ соответственно. С помощью отображения $\delta$ происходит разложение чисел в обычные цепные дроби. Для них теорема Лагранжа утверждает [2]: upрациональность допускает периодическое разложсене в иепную дробъ тогда и только тогда, когда она является квадратичной.

Приведенная выше теорема 0.1 представляет собой обобщение первой части теоремы Лагранжа для кубических иррациональностей, так как в данном случае неподвижность точки $x$ влечет за собой периодичность разложения ее в двумерную цепную дробь.

0.2. Приближения кубических единиц. В настоящей работе доказывается вторая часть обобщения теоремы Лагранжа (теорема 0.2) для некоторого трехпараметрического семейста кубических иррациональностей, точнее для точек $\alpha=\left(\alpha_{1}, \alpha_{2}\right)$ с координатами $\alpha_{1}=\theta^{2}$, $\alpha_{2}=\theta$, где $0<\theta<1-$ вещественный корень многочлена

$$
f(x)=x^{3}+a_{2} x^{2}+a_{1} x+a_{0}
$$

с коэффициентами $a_{i}$ из кольца $\mathbb{Z}$ и со свободным членом $a_{0}= \pm 1$. Такие числа $\theta$ являются единицами кубического поля $\mathbb{Q}(\theta)$. Для описанных в предложениях 4.1 и 4.2 иррациональностей $\alpha=\left(\alpha_{1}, \alpha_{2}\right)$ в теореме 8.1 доказано следующе утверждение.

ТЕОРема 0.2. 1. Существуют период $p \geqslant 1$ и такие рекуррентные последовательности $Q^{a}, R_{1}^{a}, R_{2}^{a}$ третьего порядка с постоянными коэфбичиентами, что для всех $i=a p+b$, где $0 \leqslant b<p$, точка

$$
v_{\min }^{(i)}=\left(-Q^{a} \alpha_{1}+R_{1}^{a},-Q^{a} \alpha_{2}+R_{2}^{a}\right)
$$

Исследование выполнено за счет гранта Российского научного фонда (проект № 14-11-00433). 
обладает минимальным свойством:

$$
v_{\min }^{(i)} \in T^{(i)} \quad \text { сминимальным иелым } Q^{a} \geqslant 1,
$$

где $T^{(i)}$ - явно определяемая последовательность выпукльх шестиугольников, площадь коmорых $s\left(T^{(i)}\right) \rightarrow 0$ при $i \rightarrow+\infty$. Это означает, что ни одна из точек орбиты $x_{j} \equiv$ $-j \alpha \bmod \mathbb{Z}^{2}$ не nonadaem

$$
x_{j} \notin T^{(i)} \quad \text { для всех } 1 \leqslant j<Q^{a}
$$

в шестиугольник $T^{(i)}$.

2. Для всех $a=0,1,2, \ldots$ имеет место неравенство

$$
\left|Q^{a} \alpha_{1}-R_{1}^{a}\right|+\left|Q^{a} \alpha_{2}-R_{2}^{a}\right| \leqslant c \varrho(\mathbf{A})^{a},
$$

если некоторая калибровочная $2 \times 2$-матрица $\mathbf{A}$ имеет простой спектр, где $\varrho(\mathbf{A})-$ cпектральный радиус матрицы $\mathbf{A}$ и с $>0$ - константа, не зависящая от а.

Минимальное свойство указывает на наилучшее ядерное приближение (karyon approximation). Это означает, что точки $v_{\min }^{(i)}$ наилучшим образом приближаются к $0 \bmod \mathbb{Z}^{2}$ относительно $T^{(i)}$-нормы (ядерной нормы), в качестве выпуклых тел для которой выбраны шестиугольники $T^{(i)}$ - ядра индуцированных разбиений двумерного тора $\mathbb{T}^{2}$. Данные шестиугольники $T^{(i)}$ согласованы с орбитой точек $x_{j} \equiv-j \alpha \bmod \mathbb{Z}^{2}$ для $j=0,1,2, \ldots$ и, следовательно, напрямую согласованы с интересующей нас точкой $\alpha=\left(\alpha_{1}, \alpha_{2}\right)$. Неравенства же $(0.2)$ записаны в фиксированной ромбической норме $|x|_{s}=\left|x_{1}\right|+\left|x_{2}\right|$. Можно выбрать и любую другую норму, например, $|x|=\left(\left|x_{1}\right|^{2}+\left|x_{2}\right|^{2}\right)^{1 / 2}$. Фиксированные нормы удобны для количественных оценок скорости приближений, но в их терминах не удается описывать наилучшие приближения.

В п. 9.2 показано, как неравенства (0.2) можно перенести на другие числа кубического поля $\mathbb{Q}(\theta)$, отличные от единиц. В качестве примера рассмотрена совместная аппроксимация для кубических корней $\sqrt[3]{2}, \sqrt[3]{4}$.

0.3. История и методы. Доказательство того, что приближения в теореме 0.2 являются наилучшими относительно $T^{(i)}$-норм, опирается на метод дифференцирования индуцированных разбиений многомерных торов [1]. Для вывода же количественных оценок (0.2) используются многомерные возвратные отображения [1]. Ранее одномерные возвратные отображения применялись в теории динамических систем [3], [4], а двумерные возвратные отображения для проверки периодичности разложений кубических корней [5].

Перечислим некоторые работы, в которых непосредственно рассматриваются разложения кубических иррациональностей в цепные дроби: периодичность разложений с помощью алгоритма Якоби [6], [7]; наилучшие приближения в евклидовой метрике алгоритмом Якоби-Перрона [8], [9] для корней многочленов (0.1) с коэффициентами $a_{2}=0$ и $a_{0}=-1$; а также кубические иррациональности многочленов $(0.1)$, имеющие свободный член $a_{0}=-1$ и комплексные корни [10]-[12].

\section{1. Согласованные тройки векторов и их производные}

1.1. Согласованные тройки векторов. Обозначим через $\Sigma$ совокупность всех сочетаний $\sigma$ двух элементов: $\{0,1\},\{0,2\},\{1,2\}$, из множества индексов $\{0,1,2\}$. Пусть $v_{0}, v_{1}, v_{2}-$ произвольные векторы из $\mathbb{R}^{2}, \sigma=\left\{k_{1}, k_{2}\right\}$ из $\Sigma$ и $\sigma^{\prime}=\left\{k_{3}\right\}=\{0,1,2\} \backslash \sigma$ - дополнительный к $\sigma$ индекс. Далее мы будем рассматривать неупорядоченные тройки векторов $\left\{v_{0}, v_{1}, v_{2}\right\}$, где индексы лишь обозначают векторы.

ОПРЕДЕЛЕНИЕ 1.1. Пусть $v_{k} \neq 0$ - любой вектор из $\left\{v_{0}, v_{1}, v_{2}\right\}$ и $H_{\sigma^{\prime}}$ обозначает прямую, проходящую через начало координат и имеющую направляющий вектор $v_{k_{3}}$. Тогда такое множество векторов $\left\{v_{0}, v_{1}, v_{2}\right\}$ назовем звездой, если для всех сочетаний $\sigma=\left\{k_{1}, k_{2}\right\} \in \Sigma$ векторы 
$v_{k_{1}}, v_{k_{2}}$ не принадлежат прямой $H_{\sigma^{\prime}}$ и лежат по отношению к ней в разных полуплоскостях $H_{\sigma^{\prime}}^{+}$и $H_{\sigma^{\prime}}^{-}$.

Как следствие из определения звезды вытекает линейная независимость любых двух векторов из тройки $v=\left\{v_{0}, v_{1}, v_{3}\right\}$.

Название "звезда" вытекает из следующего критерия.

КритеРий 1.1. Обозначим через $\Delta(v)$ натянутый на векторы звезды $v$ замкнутый треугольник, и пусть $\Delta^{\operatorname{int}}(v)$ - его внутренняя часть. Тогда условие на множество векторов $v$ быть звездой равносильно условию

$$
0 \in \Delta^{\mathrm{int}}(v) .
$$

1.2. Производные тройки векторов. Далее мы будем использовать обозначения

$$
X=X_{1} \sqcup X_{2}, \quad X=X_{1} \cup X_{2}
$$

для строгого и нестрогого разбиений множества $X$ в случае, если

$$
X_{1} \cap X_{2}=\varnothing \quad \text { и } \quad X_{1}^{\text {int }} \cap X_{2}^{\text {int }}=\varnothing
$$

соответственно, где $X_{k}^{\mathrm{int}}-$ множество внутренних точек из $X_{k}$.

Из определения 1.1 вытекает следующее утверждение.

Лемма 1.1. Предположим, что для некоторого сочетания $\sigma=\left\{k_{1}, k_{2}\right\}$ из $\Sigma$ сумма векторов $v_{\sigma}=v_{k_{1}}+v_{k_{2}}$ звезды $v=\left\{v_{0}, v_{1}, v_{2}\right\}$ не принадлежит прямой $H_{\sigma^{\prime}}$, где $\sigma^{\prime}-$ дополнительное сочетание для $\sigma$. Тогда при этом условии только одно из множеств

$$
v(\sigma) \sqcup v\left(\sigma^{\prime}\right)
$$

будет звездой. Здесъ $v(\sigma)=\left\{v_{k_{1}}, v_{\sigma}\right\}$ или $v(\sigma)=\left\{v_{\sigma}, v_{k_{2}}\right\}$ в зависимости от того, какие из пар векторов $v_{k_{1}}, v_{\sigma}$ или $v_{k_{2}}, v_{\sigma}$ принадлежат разным полуплоскостям $H_{\sigma^{\prime}}^{ \pm}, u v\left(\sigma^{\prime}\right)-$ дополнительный для $v(\sigma)$ вектор из звезды $v$.

ОПРЕДЕЛЕНИЕ 1.2. Обозначим через $v^{\sigma}=v(\sigma) \sqcup v\left(\sigma^{\prime}\right)$ то множество векторов из (1.3), которое образует звезду. Если существуют множества векторов $v^{\sigma}$ для всех сочетаний $\sigma \in \Sigma$, т.е. для всех $\sigma$ выполняется условие леммы 1.1 , то будем говорить, что звезда $v=\left\{v_{0}, v_{1}, v_{2}\right\}$ невырожденна.

Таким образом, согласно определению 1.2 для всех сочетаний $\sigma=\left\{k_{1}, k_{2}\right\}$ из $\Sigma$ на множестве невырожденных звезд $v=\left\{v_{0}, v_{1}, v_{2}\right\}$ определено отображение

$$
v \stackrel{\sigma}{\rightarrow} v^{\sigma}=\left\{v_{0}^{\sigma}, v_{1}^{\sigma}, v_{2}^{\sigma}\right\}
$$

где $v_{k_{1}}^{\sigma}=v_{k_{1}}, v_{k_{2}}^{\sigma}=v_{\sigma}$ или $v_{k_{1}}^{\sigma}=v_{\sigma}, v_{k_{2}}^{\sigma}=v_{k_{2}}$ выбираются в согласии с $(1.3)$ и $v_{k^{\prime}}^{\sigma}=v_{k^{\prime}}$ для остальных $k^{\prime} \in \sigma^{\prime}$.

Звезду $v^{\sigma}$ из (1.4) назовем $\sigma$-производной невырожденной звезды $v$. Если нужно выделить индексы $k_{1}, k_{2}$ в сочетании $\sigma=\left\{k_{1}, k_{2}\right\}$, то будем для $\sigma$-производной (1.4) использовать еще и другое развернутое обозначение $v^{\sigma}=v^{\left\{k_{1}, k_{2}\right\}}$.

\section{2. Индуцированные разбиения тора}

2.1. Перекладывающиеся развертки тора. Пусть $L=\mathbb{Z}\left[l_{1}, l_{2}\right]$ - полная решетка в $\mathbb{R}^{2}$ с базисом $l_{1}, l_{2}$, т.е. векторы $l_{1}, l_{2}$ линейно независимы на полем вещественных чисел $\mathbb{R}$, и пусть $T$ - некоторое подмножество из $\mathbb{R}^{2}$. Будем говорить, что $T$ является разверткой тора 
$\mathbb{T}_{L}^{2}=\mathbb{R}^{2} / L$, если отображение $T \rightarrow \mathbb{T}_{L}^{2}: x \mapsto x \bmod L-$ биекция. Развертка $T$ называется перекладъвающейся, если заданы ее разбиение

$$
T=T_{0} \sqcup T_{1} \sqcup T_{2}
$$

и перекладывание

$$
T \stackrel{S^{\prime}}{\longrightarrow} T: S^{\prime}(x)=x+v_{\operatorname{col}(x)}
$$

на векторы $v_{0}, v_{1}, v_{2}$, связанные с базисом решетки $L$ равенствами

$$
l_{k}=v_{k}-v_{0} \quad \text { для } \quad k=1,2 .
$$

Здесь использовано обозначение $\operatorname{col}(x)=k$ для цвета точек $x$, принадлежащих подмножеству $T_{k}$, где $k=0,1,2$. Заметим, что при переходе (2.2) от векторов переклыдывания $v_{0}, v_{1}$, $v_{2}$ к базизу $l_{1}, l_{2}$ решетки $L$ нарушается симметрия, когда выделяется вектор $v_{0}$. Удобно для него ввести дополнительное обозначение

$$
v_{0}=\alpha^{\prime}
$$

В частности, из равенств (2.2) и (2.3) вытекают сравнения $v_{k} \equiv \alpha^{\prime} \bmod L$ для всех $k=0,1,2$. Поэтому перекладывание $S^{\prime}$ представляет собой сдвиг тора $S^{\prime}=S_{\alpha^{\prime}}^{\prime}$

$$
T \stackrel{S^{\prime}}{\longrightarrow} T: S^{\prime}(x) \equiv x+\alpha^{\prime} \bmod L
$$

на вектор $\alpha^{\prime} \bmod L$

2.2. Шестиугольная развертка тора. Обозначим через $T_{k_{1}, k_{2}}$ замкнутый параллелограмм, натянутый на векторы $v_{k_{1}}, v_{k_{2}}$. Определим для $m=0,1,2$ параллелограммы $T_{m}$, имеющие те же внутренние части $T_{m}^{\text {int }}=T_{k, l}^{\text {int }}$, что и параллелограммы $T_{k_{1}, k_{2}}$, где $m$ - дополнительный к $\left\{k_{1}, k_{2}\right\}$ индекс в $\{0,1,2\}$. Стороны и вершины параллелограммов $T_{k_{1}, k_{2}}$ распределим между соответствующими параллелограммами $T_{m}$ так, чтобы выполнялись следующие условия:

1) каждому $T_{m}$ принадлежат две смежные стороны и соединяющая их вершина;

2) получающееся при этом объединение $T=T_{0} \cup T_{1} \cup T_{2}$ является разбиением - нестрогим разбиением (1.2) - множества $T$.

Для определенности положим

$$
0 \in T_{0}, \quad v_{0} \in T_{1}, \quad v_{0}+v_{1} \in T_{2} .
$$

В результате получается шестиугольник

$$
T=T(v)=T_{0} \sqcup T_{1} \sqcup T_{2}
$$

с попарно равными и параллельными сторонами. Такой шестиугольник будет перекладывающейся разверткой тора $\mathbb{T}_{L}^{2}$ для решетки $L$ с базисом $(2.2)$ и векторами перекладывания $v_{0}$, $v_{1}, v_{2}$ в случае, если данная тройка векторов $v=\left\{v_{0}, v_{1}, v_{2}\right\}$ образует звезду.

2.3. Вмещающее пространство. Кроме тора $\mathbb{T}_{L}^{2}$ нам потребуется еще один стандартный тор $\mathbb{T}^{2}=\mathbb{R}^{2} / \mathbb{Z}^{2}$ для квадратной решетки $\mathbb{Z}^{2}=\mathbb{Z}\left[e_{1}, e_{2}\right]$ с ортонормированным базисом $\left\{e_{1}, e_{2}\right\}$.

Зададим сдвиг $S=S_{\alpha}$ тора $\mathbb{T}^{2}$ на вектор $\alpha=\left(\alpha_{1}, \alpha_{2}\right)$ из $\mathbb{R}^{2}$, полагая

$$
\mathbb{T}^{2} \stackrel{S}{\longrightarrow} \mathbb{T}^{2}: x \mapsto S(x) \equiv x+\alpha \bmod \mathbb{Z}^{2} .
$$

Далее торы $\mathbb{T}^{2}$ будут использоваться как вмещающие пространства для вложений различных торов $\mathbb{T}_{L}^{2}$ с изменяющимися решетками $L$. 
2.4. Вкладывающиеся в тор развертки. Подмножество $T \subset \mathbb{R}^{2}$ называется $\mathbb{Z}^{2}$-различимым, т.е. для любых элементов $x, y$ из $T$, связанных сравнением $x \equiv y \bmod \mathbb{Z}^{2}$, следует их равенство $x=y$.

ОПРЕДЕЛЕНиЕ 2.1. Перекладывающаяся развертка $T$ из (2.1) вкладывается

$$
T \stackrel{\text { em }}{\hookrightarrow} \mathbb{T}^{2}
$$

в тор $\mathbb{T}^{2}$ относительно сдвига $S=S_{\alpha}$, если выполняются следующие условия:

1) подмножество $T \subset \mathbb{R}^{2}$ является $\mathbb{Z}^{2}$-различимым и, значит, отображение

$$
T \rightarrow T \bmod \mathbb{Z}^{2}: x \mapsto x \bmod \mathbb{Z}^{2}
$$

есть биекция; поэтому, используя отображение (2.9), можем считать развертку $T$ вложенной как множество

$$
T \subset \mathbb{T}^{2}
$$

в тор $\mathbb{T}^{2}$

2) векторы перекладывания имеют вид

$$
v_{k} \equiv m_{k} \alpha \bmod \mathbb{Z}^{2}
$$

для всех $k=0,1,2$ с некоторыми коэффициентами $m_{k}=1,2,3, \ldots$;

3) орбиты

$$
\operatorname{Orb}^{\prime}\left(T_{k}\right)=\left\{S^{j}\left(T_{k}\right): j=1, \ldots, m_{k}-1\right\}
$$

подмножеств $T_{k} \subset T$ удовлетворяют условию

$$
\operatorname{Orb}^{\prime}\left(T_{k}\right) \cap T=\varnothing
$$

для $k=0,1,2$.

Чтобы сформулировать следующий результат, нам потребуется в дополнение к (2.12) определить еще полные орбиты

$$
\operatorname{Orb}\left(T_{k}\right)=\left\{S^{j}\left(T_{k}\right): j=0,1, \ldots, m_{k}-1\right\}
$$

Кроме того, будем предполагать вектор сдвига $\alpha$ из (2.7) ирращиональным, когда

$$
1, \alpha_{1}, \alpha_{2} \text { линейно независимы над кольцом } \mathbb{Z} \text {. }
$$

В [13] доказана следующая теорема.

Теорема 2.1. Пусть развертка $T$ вкладывается (2.8) в тор $\mathbb{T}^{2}$, развертка $T$ имеет внутреннюю точку, и пусть вектор $\alpha$ сдвига $S=S_{\alpha}$ из (2.7) будет иррациональным (2.15). Тогда выполняются следующие утверждения:

1) множества из полных орбит $\operatorname{Orb}\left(T_{k}\right)$ не пересекаются, m.е.

$$
S^{j_{1}}\left(T_{k_{1}}\right) \cap S^{j_{2}}\left(T_{k_{2}}\right) \neq \varnothing
$$

только при условии $j_{1}=j_{2}$ u $k_{1}=k_{2}$;

2) имеет место разбиение тора $\mathbb{T}^{2}$ :

$$
\mathcal{T}=\mathcal{T}_{0} \sqcup \mathcal{T}_{1} \sqcup \mathcal{T}_{2}, \quad \text { əде } \quad \mathcal{T}_{k}=T_{k} \sqcup S^{1}\left(T_{k}\right) \sqcup \cdots \sqcup S^{m_{k}-1}\left(T_{k}\right)
$$

- орбитное разбиение, составленное из множеств, входящих в орбиту $\operatorname{Orb}\left(T_{k}\right)$ из $(2.14)$. 
2.5. Индуцированные отображения и ядро разбиения. Из теоремы 2.1 следует, что сдвиг тора $S^{\prime}: T \rightarrow T$ из (2.4) является индуиированным отображением, или, иначе, отображением первого возвращения в $T \subset \mathbb{T}^{2}$, отображением Пуанкаре - для сдвига тора $S: \mathbb{T}^{2} \rightarrow \mathbb{T}^{2}$ из $(2.7)$, что символически будем обозначать в виде равенства $S^{\prime}=\left.S\right|_{T}$. Обозначим соответственно через

$$
T=T(v) \quad \text { и } \quad \mathcal{T}=\mathcal{T}(v)=\mathcal{T}_{0} \sqcup \mathcal{T}_{1} \sqcup \mathcal{T}_{2}
$$

развертку $T$ из (2.1) и индуцированное разбиение тора $\mathbb{T}^{2}$, порождаемое вкладывающейся в тор $T \stackrel{\text { еm }}{\hookrightarrow} \mathbb{T}^{2}$ разверткой $T$. Множество $T$ по отношению ко всему разбиению тора $\mathcal{T}$ называется [14] ядром (karyon) разбиения $\mathcal{T}$. Чтобы указывать на такую связь между $T$ и $\mathcal{T}$, будем использовать обозначение

$$
T=\operatorname{Kr}=\operatorname{Kr}(\mathcal{T}) .
$$

Ядро $\mathrm{Kr}$ характеризуется следующим свойством: $\mathrm{Kr}$ - это такое подмножество $\mathrm{Kr} \subset \mathbb{T}^{2}$, для которого отображение первого возвращения $S^{\prime}=\left.S\right|_{\mathrm{Kr}}$, индуцированное сдвигом тора $S=S_{\alpha}$ из (2.7), эквивалентно перекладыванию трех подмножеств из разбиения

$$
\mathrm{Kr}=\mathrm{Kr}_{0} \sqcup \mathrm{Kr}_{1} \sqcup \mathrm{Kr}_{2} .
$$

Понятие ядра $\mathrm{Kr}$ является основным для всей рассматриваемой здесь конструкции. С помощью ядер $T=\mathrm{Kr}$ будут построены наилучшие приближений для кубических иррациональностей; они же будут задавать ядерные $T$-нормы, используемые для получения количественных оценок скорости приближений.

2.6. Критерий вложимости шестиугольной развертки тора. Пусть $T=T(v)$ будет некоторой разверткой (2.1), которая изначально не предполагается вложенной в тор $\mathbb{T}^{2}$; и пусть (см. соглашение (1.2))

$$
\mathcal{T}=\mathcal{T}(v)=\mathcal{T}_{0} \cup \mathcal{T}_{1} \cup \mathcal{T}_{2}
$$

- подмножество тора $\mathbb{T}^{2}$, определеное по правилу из (2.16). Таким образом, в данном случае множество (2.17) не обязательно является разбиением тора $\mathbb{T}^{2}$.

В [13] доказана следующая теорема.

Теорема 2.2. Развертка $T=T(v)$ вкладьвается (2.8) в тор $T \stackrel{\text { em }}{\hookrightarrow} \mathbb{T}^{2}$ тогда и только тогда, когда выполняется одно из следующих двух эквивалентных утверждений:

1) определенное в (2.17) множество $\mathcal{T}=\mathcal{T}(v)$ является разбиением тора $\mathbb{T}^{2}$;

2) внутренняя часть $T^{\mathrm{int}}$ развертки $T \subset \mathbb{T}^{2}$ не содержит ни одной из точек $x_{j}$ орбить

$$
\operatorname{Orb}^{\prime}(0, \mathbf{m})=\left\{x_{j}=S^{j}(0): j=1,2, \ldots, \mathbf{m}-1\right\},
$$

əде $\mathbf{m}=m_{0}+m_{1}+m_{2}$.

\section{7. Производные вкладывающихся троек векторов.}

ОПРЕДЕЛЕНИЕ 2.2. Пусть $v=\left\{v_{0}, v_{1}, v_{2}\right\}$ - звезда и $T=T(v)$ - отвечающая ей развертка (2.16) тора $\mathbb{T}_{L}^{2}$ с векторами перекладывания $v_{0}, v_{1}, v_{2}$. Если данная развертка $T$ вкладывается в тор $\mathbb{T}^{2}$ :

$$
T \stackrel{e m}{\longleftrightarrow} \mathbb{T}^{2}
$$

относительно некоторого сдвига $S=S_{\alpha}$, то в этом случае будем говорить, что такая звезда $v$ вкладывается

$$
v \stackrel{\mathrm{em}}{\hookrightarrow} \mathbb{T}^{2}
$$

в тор $\mathbb{T}^{2}$ относительно сдвига $S$. 
В [15] доказана следующая теорема.

ТЕОрема 2.3. Пусть невырожденная звезда $v=\left\{v_{0}, v_{1}, v_{2}\right\}$ вкладывается (2.18) в тор $\mathbb{T}^{2}$

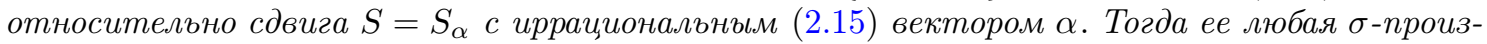
водная $v^{\sigma}=\left\{v_{0}^{\sigma}, v_{1}^{\sigma}, v_{2}^{\sigma}\right\}$ для $\sigma \in \Sigma$ также вкладывается

$$
v^{\sigma} \stackrel{\mathrm{em}}{\hookrightarrow} \mathbb{T}^{2}
$$

в тот же тор $\mathbb{T}^{2}$ относительно сдвига $S$.

\section{3. Возвратные отображения}

3.1. Базисный треугольник. Основной областью для нас будет замкнутый треугольник $\triangle$ с вершинами в точках $(0,0),(1,0)$ и $(0,1)$. Обозначим через $S_{\triangle}$ группу его аффинных симметрий (ангармоническая группа). Она сопряжена с группой метрических симметрий правильного треугольника и состоит из трех аффинных поворотов $r_{0}, r_{1}, r_{2}$, соответствующих поворотам против часовой стрелки на $0^{\circ}, 120^{\circ}, 240^{\circ}$, и трех осевых симметрий $h_{0}, h_{1}, h_{2}$ относительно медиан $\mu_{0}, \mu_{1}, \mu_{2}$, проходящих через вершины $0=(0,0), 1=(1,0), 2=(0,1)$ соответственно. В координатах $\left(x_{1}, x_{2}\right) \in \triangle$ относительно декартова базиса $e_{1}=(1,0), e_{2}=(0,1)$ симметрии из $S_{\triangle}$ записываются следующим образом:

$$
\begin{array}{ll}
r_{0}\left(x_{1}, x_{2}\right)=\left(x_{1}, x_{2}\right), & h_{0}\left(x_{1}, x_{2}\right)=\left(x_{2}, x_{1}\right), \\
r_{1}\left(x_{1}, x_{2}\right)=\left(-x_{1}-x_{2}+1, x_{1}\right), & h_{1}\left(x_{1}, x_{2}\right)=\left(x_{1},-x_{1}-x_{2}+1\right), \\
r_{2}\left(x_{1}, x_{2}\right)=\left(x_{2},-x_{1}-x_{2}+1\right), & h_{2}\left(x_{1}, x_{2}\right)=\left(-x_{1}-x_{2}+1, x_{2}\right) .
\end{array}
$$

3.2. Разбиения треугольника. Выделим в треугольнике $\triangle$ шесть открытых областей $\triangle_{k}^{k l}, \triangle_{l}^{k l} \subset \triangle$ с целыми индексами $0 \leqslant k<l \leqslant 2$. Замыкания областей $\bar{\triangle}_{k}^{k l}, \bar{\triangle}_{l}^{k l}$ разбивают треугольник: $\triangle=\bar{\triangle}_{k}^{k l} \cup \bar{\triangle}_{l}^{k l}$, и пересекаются по медиане $\mu_{m}: \bar{\triangle}_{k}^{k l} \cap \bar{\triangle}_{l}^{k l}=\mu_{m}$, с номером $m$, дополняющим индексы $\{k, l\}$ до всего множества $\{0,1,2\}$. Нижние индексы в $\triangle_{k}^{k l}$ и $\triangle_{l}^{k l}$ указывают на принадлежность вершин с номерами $k$ и $l$ соответственно $\bar{\triangle}_{k}^{k l}$ и $\bar{\triangle}_{l}^{k l}$. Таким образом, области $\triangle_{k}^{k l}, \triangle_{l}^{k l}$ представляют собой открытые треугольники, замыкание объединения которых

$$
\triangle^{k l}=\triangle_{k}^{k l} \sqcup \triangle_{l}^{k l}
$$

совпадает со всем треугольником $\triangle$ и при этом $\triangle_{k}^{k l} \cap \triangle_{l}^{k l}=\varnothing$.

3.3. Нормированные дифференцирования звезд. Согласно условию (1.1) из критерия 1.1 каждая точка $x \in \triangle^{k l}$ задает звезду $w=\left\{w_{0}, w_{1}, w_{2}\right\}$ с лучами

$$
w_{0}=-x, \quad w_{1}=e_{1}-x, \quad w_{2}=e_{2}-x,
$$

выходящими из центра $x$ в вершины с соответствующими номерами $0,1,2$. В этом случае существует производная звезда

$$
w^{\prime}=\left\{w_{0}^{\prime}, w_{1}^{\prime}, w_{2}^{\prime}\right\}
$$

Если $x \in \triangle_{k}^{k l}$, то лучи в (3.4) имеют вид $w_{m}^{\prime}=w_{m}, w_{k}^{\prime}=w_{k}, w_{l}^{\prime}=w_{k}+w_{l}$; если же $x \in \triangle_{l}^{k l}$, то вид $w_{m}^{\prime}=w_{m}, w_{k}^{\prime}=w_{k}+w_{l}, w_{l}^{\prime}=w_{l}$. Здесь мы использовали более удобное для дальнейшего обозначение для производной $w^{\prime}$ звезды $w$ вместо ранее введенного $w^{\sigma}$ в $(1.4)$; индекс $m$ снова дополняет $\{k, l\}$ до всего множества индексов $\{0,1,2\}$.

Из условия $x \in \triangle^{k l}$ вытекает, что векторы $e_{1}^{\prime}=v_{1}^{\prime}-v_{0}^{\prime}, e_{2}^{\prime}=v_{2}^{\prime}-v_{0}^{\prime}$ образуют базис пространства $\mathbb{R}^{2}$. Пусть $A^{k l}$ - матрица перехода $e^{\prime}=e A^{k l}$ от базиса $\left\{e_{1}, e_{2}\right\}$ к базису $\left\{e_{1}^{\prime}, e_{2}^{\prime}\right\}$, где 
$e^{\prime}=\left(e_{1}^{\prime} e_{2}^{\prime}\right)$ - строка, $e A^{k l}$ - произведение строки $e=\left(e_{1} e_{2}\right)$ на $2 \times 2$-матрицу $A^{k l}$. Данная матрица имеет две специализащии $A^{k l}=A_{k}^{k l}$ или $A^{k l}=A_{l}^{k l}$ в зависимости от принадлежности $x$ области $\triangle_{k}^{k l}$ или $\triangle_{l}^{k l}$ :

$$
\begin{aligned}
A_{1}^{12} & =\left(\begin{array}{ll}
1 & 1-x_{1} \\
0 & 1-x_{2}
\end{array}\right), & A_{2}^{12} & =\left(\begin{array}{cc}
1-x_{1} & 0 \\
1-x_{2} & 1
\end{array}\right), \\
A_{0}^{01} & =\left(\begin{array}{cc}
1-x_{1} & 0 \\
-x_{2} & 1
\end{array}\right), & A_{1}^{01} & =\left(\begin{array}{cc}
x_{1} & -1+x_{1} \\
x_{2} & 1+x_{2}
\end{array}\right), \\
A_{0}^{02} & =\left(\begin{array}{cc}
1 & -x_{1} \\
0 & 1-x_{2}
\end{array}\right), & A_{2}^{02} & =\left(\begin{array}{cc}
1+x_{1} & x_{1} \\
-1+x_{2} & x_{2}
\end{array}\right) .
\end{aligned}
$$

В координатах формула перехода $e^{\prime}=e A^{k l}$ примет вид

$$
\left(\begin{array}{l}
x_{1}^{\prime} \\
x_{2}^{\prime}
\end{array}\right)=\mathcal{A}^{k l}\left(\begin{array}{l}
x_{1} \\
x_{2}
\end{array}\right) .
$$

Здесь, чтобы не усложнять обозначения, обратные матрицы $\left(A^{k l}\right)^{-1}$ для $A^{k l}$ обозначены через $\mathcal{A}^{k l}$ :

$$
\begin{array}{rlrl}
\mathcal{A}_{1}^{12} & =\frac{1}{1-x_{2}}\left(\begin{array}{cc}
1-x_{2} & -1+x_{1} \\
0 & 1
\end{array}\right), & \mathcal{A}_{2}^{12}=\frac{1}{1-x_{1}}\left(\begin{array}{cc}
1 & 0 \\
-1+x_{2} & 1-x_{1}
\end{array}\right), \\
\mathcal{A}_{0}^{01}=\frac{1}{1-x_{1}}\left(\begin{array}{cc}
1 & 0 \\
x_{2} & 1-x_{1}
\end{array}\right), & \mathcal{A}_{1}^{01}=\frac{1}{x_{1}+x_{2}}\left(\begin{array}{cc}
1+x_{2} & 1-x_{1} \\
-x_{2} & x_{1}
\end{array}\right), \\
\mathcal{A}_{0}^{02}=\frac{1}{1-x_{2}}\left(\begin{array}{cc}
1-x_{2} & x_{1} \\
0 & 1
\end{array}\right), & \mathcal{A}_{2}^{02}=\frac{1}{x_{1}+x_{2}}\left(\begin{array}{cc}
x_{2} & -x_{1} \\
1-x_{2} & 1+x_{1}
\end{array}\right) .
\end{array}
$$

$\mathrm{C}$ помощью обратных матриц $\mathcal{A}^{k l}$ можно для производной звезды $w^{\prime}$ из (3.4) определить нормированную звезду

$$
\mathbf{w}^{\prime}=\left\{\mathbf{w}_{0}^{\prime}, \mathbf{w}_{1}^{\prime}, \mathbf{w}_{2}^{\prime}\right\}
$$

с центром $x^{\prime}=\left(x_{1}^{\prime}, x_{2}^{\prime}\right)$, вычисляемым по формуле

$$
\left(\begin{array}{l}
x_{1}^{\prime} \\
x_{2}^{\prime}
\end{array}\right)=\mathcal{A}^{k l}\left(\begin{array}{l}
-w_{01}^{\prime} \\
-w_{02}^{\prime}
\end{array}\right),
$$

где в правом столбце использованы координаты вектора $w_{0}^{\prime}=\left(w_{01}^{\prime}, w_{02}^{\prime}\right)$ из производной звезды $w^{\prime}=\left\{w_{0}^{\prime}, w_{1}^{\prime}, w_{2}^{\prime}\right\}$, определнной в (3.4). Если вектор $w_{0}^{\prime}$ сохраняется неизменным, $w_{0}^{\prime}=w_{0}$, то в формуле (3.6) выбирается центр $x=\left(x_{1}, x_{2}\right)=-w_{0}=-w_{0}^{\prime}$ первоначальной звезды $w$. Нормированная звезда $\mathbf{w}^{\prime}$, как и $w$, имеет лучи

$$
\mathbf{w}_{0}^{\prime}=-x^{\prime}, \quad \mathbf{w}_{1}^{\prime}=e_{1}-x^{\prime}, \quad \mathbf{w}_{2}^{\prime}=e_{2}-x^{\prime},
$$

выходящие теперь уже из нового центра $x^{\prime}$ в вершины треугольника $\triangle$. Определение $(3.8)$ звезды $\mathbf{w}^{\prime}$ корректно, поскольку по определению (2.10) точка $x^{\prime}$ принадлежит внутренней области $\triangle^{\text {int }}$ треугольника $\triangle$.

В явном виде координаты $\left(x_{1}^{\prime}, x_{2}^{\prime}\right)=\delta^{k l}\left(x_{1}, x_{2}\right)$ центра $x^{\prime}$ нормированной звезды $\mathbf{w}^{\prime}$ из $(3.8)$ вычисляются через дробно-линейные преобразования:

$$
\begin{aligned}
\delta_{1}^{12}\left(x_{1}, x_{2}\right) & =\left(\frac{x_{1}-x_{2}}{1-x_{2}}, \frac{x_{2}}{1-x_{2}}\right), & \delta_{2}^{12}\left(x_{1}, x_{2}\right) & =\left(\frac{x_{1}}{1-x_{1}}, \frac{-x_{1}+x_{2}}{1-x_{1}}\right), \\
\delta_{0}^{01}\left(x_{1}, x_{2}\right) & =\left(\frac{x_{1}}{1-x_{1}}, \frac{x_{2}}{1-x_{1}}\right), & \delta_{1}^{01}\left(x_{1}, x_{2}\right) & =\left(\frac{2 x_{1}+x_{2}-1}{x_{1}+x_{2}}, \frac{x_{2}}{x_{1}+x_{2}}\right), \\
\delta_{0}^{02}\left(x_{1}, x_{2}\right) & =\left(\frac{x_{1}}{1-x_{2}}, \frac{x_{2}}{1-x_{2}}\right), & \delta_{2}^{02}\left(x_{1}, x_{2}\right) & =\left(\frac{x_{1}}{x_{1}+x_{2}}, \frac{x_{1}+2 x_{2}-1}{x_{1}+x_{2}}\right) .
\end{aligned}
$$

Преобразования (3.10) представляют собой двумерный аналог одномерных возвратных отображений [3], [4]. 
3.4. Возвратные отображения. Дробно-линейные преобразования (3.10) задают три отображения $\delta^{k l}$ :

$$
\triangle \stackrel{\delta^{k l}}{\longrightarrow} \triangle: x \mapsto x^{\prime}=\delta^{k l}(x),
$$

с индексами $0 \leqslant k<l \leqslant 2: \delta^{k l}(x)=\delta_{k}^{k l}(x)$, если $x \in \triangle_{k}^{k l}$, и $\delta^{k l}(x)=\delta_{l}^{k l}(x)$, если $x \in$ $\triangle_{l}^{k l}$. Таким образом, областью определения отображения $\delta^{k l}$ является открытая двусвязная область $\triangle^{k l} \subset \triangle$ из (3.2) и, значит, $\delta^{k l}$ определены почти всюду в треугольнике $\triangle$, исключая его границы и медиану $\mu_{m}$, проходящую через вершину с номером $m$ из дополнения $\{0,1,2\} \backslash$ $\{k, l\}$.

3.5. Матрицы возвратных отображений. На языке $3 \times 3$-матриц

$$
M=\left(\begin{array}{lll}
a_{11} & a_{12} & a_{13} \\
a_{21} & a_{22} & a_{23} \\
a_{31} & a_{32} & a_{33}
\end{array}\right)
$$

дробно-линейные преобразования (3.10) записываются в виде

$$
M\langle x\rangle=\left(\frac{a_{11} x_{1}+a_{12} x_{2}+a_{13}}{a_{31} x_{1}+a_{32} x_{2}+a_{33}}, \frac{a_{21} x_{1}+a_{22} x_{2}+a_{23}}{a_{31} x_{1}+a_{32} x_{2}+a_{33}}\right)
$$

для $x=\left(x_{1}, x_{2}\right)$. В матричной форме (3.12) удобно представляется ассоциативное свойство таких преобразований

$$
M_{1}\left\langle M_{2}\langle x\rangle\right\rangle=\left(M_{1} \cdot M_{2}\right)\langle x\rangle .
$$

Поскольку $E_{3}\langle x\rangle=x$ для единичной матрицы $E_{3}$ порядка 3 , то из свойства (3.13) следует, что для $x^{\prime}=M\langle x\rangle$ обратным отображением будет $x=M^{-1}\left\langle x^{\prime}\right\rangle$.

Согласно определению (3.12) дробно-линейные преобразования (3.10) имеют следующие матрицы:

$$
\begin{array}{rlrl}
\widehat{\delta}_{1}^{12} & =\left(\begin{array}{ccc}
1 & -1 & 0 \\
0 & 1 & 0 \\
0 & -1 & 1
\end{array}\right), & \widehat{\delta}_{2}^{12}=\left(\begin{array}{ccc}
1 & 0 & 0 \\
-1 & 1 & 0 \\
-1 & 0 & 1
\end{array}\right), \\
\widehat{\delta}_{0}^{01}=\left(\begin{array}{ccc}
1 & 0 & 0 \\
0 & 1 & 0 \\
-1 & 0 & 1
\end{array}\right), & \widehat{\delta}_{1}^{01}=\left(\begin{array}{ccc}
2 & 1 & -1 \\
0 & 1 & 0 \\
1 & 1 & 0
\end{array}\right), \\
\widehat{\delta}_{0}^{02}=\left(\begin{array}{ccc}
1 & 0 & 0 \\
0 & 1 & 0 \\
0 & -1 & 1
\end{array}\right), & \widehat{\delta}_{2}^{02}=\left(\begin{array}{ccc}
1 & 0 & 0 \\
1 & 2 & -1 \\
1 & 1 & 0
\end{array}\right) .
\end{array}
$$

Матрицы (3.14) принадлежат группе унимодулярных матрии, $\mathrm{GL}_{3}(\mathbb{Z})$.

3.6. Матрицы симметрий треугольника. Симметрии треугольника (3.1) также можно представить в матричной форме (3.12). Для них матрицами будут: для поворотов -

$$
\widehat{r}_{0}=\left(\begin{array}{ccc}
1 & 0 & 0 \\
0 & 1 & 0 \\
0 & 0 & 1
\end{array}\right), \quad \widehat{r}_{1}=\left(\begin{array}{ccc}
-1 & -1 & 1 \\
1 & 0 & 0 \\
0 & 0 & 1
\end{array}\right), \quad \widehat{r}_{2}=\left(\begin{array}{ccc}
0 & 1 & 0 \\
-1 & -1 & 1 \\
0 & 0 & 1
\end{array}\right) ;
$$

для осевых симметрий -

$$
\widehat{h}_{0}=\left(\begin{array}{lll}
0 & 1 & 0 \\
1 & 0 & 0 \\
0 & 0 & 1
\end{array}\right), \quad \widehat{h}_{1}=\left(\begin{array}{ccc}
1 & 0 & 0 \\
-1 & -1 & 1 \\
0 & 0 & 1
\end{array}\right), \quad \widehat{h}_{2}=\left(\begin{array}{ccc}
-1 & -1 & 1 \\
0 & 1 & 0 \\
0 & 0 & 1
\end{array}\right) .
$$

Матрицы симметрий треугольника (3.15), (3.16) отличаются от матриц возвратных отображений (3.14) нижней строкой $\left(\begin{array}{lll}0 & 0 & 1\end{array}\right)$. Матрицы поворотов имеют определитель $\left|\widehat{r}_{k}\right|=1$, 
а осевых симметрий - определитель $\left|\widehat{h}_{k}\right|=-1$, что отражает отличие собственных преобразований $r_{k}$ от несобственных $h_{k}$. Кроме самих матриц $(3.15),(3.16)$ нам потребуются еще их однородные части - верхние левые $(2 \times 2)$-блоки:

$$
\begin{array}{lll}
\breve{r}_{0}=\left(\begin{array}{ll}
1 & 0 \\
0 & 1
\end{array}\right), & \breve{r}_{1}=\left(\begin{array}{cc}
-1 & -1 \\
1 & 0
\end{array}\right), & \breve{r}_{2}=\left(\begin{array}{cc}
0 & 1 \\
-1 & -1
\end{array}\right), \\
\check{h}_{0}=\left(\begin{array}{ll}
0 & 1 \\
1 & 0
\end{array}\right), & \breve{h}_{1}=\left(\begin{array}{cc}
1 & 0 \\
-1 & -1
\end{array}\right), & \breve{h}_{2}=\left(\begin{array}{cc}
-1 & -1 \\
0 & 1
\end{array}\right) .
\end{array}
$$

Матрицы (3.17) порождают группу $S_{\searrow}$ аффинных (однородных) симметрий треугольника $\triangle$, получающегося сдвигом треугольника $\triangle$ на вектор $(-1 / 3,-1 / 3)$. Следовательно, треугольник $\asymp$ имеет центр в начале координат $(0,0)$ - неподвижной точке всех симметрий $(3.17)$.

3.7. Иррациональные точки. Аналогично определению (2.15) для векторов точку $x=$ $\left(x_{1}, x_{2}\right)$ из $\mathbb{R}^{2}$ назовем иррациональной, если

$$
1, x_{1}, x_{2} \text { линейно независимы над кольцом } \mathbb{Z} .
$$

Лемма 3.1 [1]. Если точка $x=\left(x_{1}, x_{2}\right)$ иррациональна (3.18) и матрица $M$ принадлежит иелочисленной унимодулярной группе $\mathrm{GL}_{3}(\mathbb{Z})$, то:

1) для точки $x$ определен ее образ $M\langle x\rangle$ относительно дробно-линейного отображения $(3.12)$;

2) соответствующая точка $x^{\prime}=M\langle x\rangle$ снова будет иррациональной.

\section{4. Разложение в произведение элементарных возвратных матриц}

4.1. Кубические единицы. Пусть

$$
f(x)=x^{3}+a_{2} x^{2}+a_{1} x+a_{0}
$$

будет многочленом с коэффициентами $a_{i}$ из кольца целых рациональных чисел $\mathbb{Z}$ и со свободным членом $a_{0}= \pm 1$. Будем говорить, что $f(x)$ является $\triangle$-многочленом, если $f(x)$ имеет вещественный корень $f(\theta)=0$, удовлетворяющий условию

$$
\alpha=\left(\alpha_{1}, \alpha_{2}\right) \in \triangle^{\mathrm{int}},
$$

где $\alpha_{1}=\theta^{2}, \alpha_{2}=\theta$. Если при этом $\theta$ - кубическая иррациональность, то скажем, что $f(x)-$ $\triangle_{\mathrm{irr}}$-многочлен, а точка $\alpha$ удовлетворяют $\triangle_{\mathrm{irr}}$-условию. Значит, в данном случае $f(x)$ будет многочленом, неприводимым над полем рациональных чисел $\mathbb{Q}$. Из $\triangle_{\text {irr }}$-условия и ограничения на свободный член $a_{0}= \pm 1$ многочлена (4.1) вытекает, что его корень $\theta$ является единицей вещественного кубического поля $\mathbb{Q}(\theta)$.

4.2. Возвратные матрицы для кубических единиц. Каждому набору $a=\left(a_{2}, a_{1}, a_{0}\right)$ коэффициентов из (4.1) сопоставим матрицу

$$
M(a)=\left(\begin{array}{ccc}
-a_{2} & -a_{1} & -a_{0} \\
1 & 0 & 0 \\
0 & 1 & 0
\end{array}\right) .
$$

Так как $\operatorname{det} M(a)=-a_{0}=\mp 1$, то $M(a)$ принадлежит группе унимодулярных матриц $\mathrm{GL}_{3}(\mathbb{Z})$. Из определения (4.3) следует, что столбец $\widehat{\alpha}=\left(\begin{array}{lll}\alpha_{1} & \alpha_{2} & 1\end{array}\right)^{T}$ является собственным вектором матрицы $M(a)$, т.е. выполняется матричное равенство

$$
M(a) \widehat{\alpha}=\lambda \widehat{\alpha}
$$

с собственным значением $\lambda=\theta$. 
4.3. Полугруппа $\widehat{\mathcal{D}}$. Обозначим через $\widehat{\mathcal{D}}$ полугруппу унимодулярных матриц из $\mathrm{GL}_{3}(\mathbb{Z})$, отвечающих преобразованиям $\delta$ из полугруппы $\mathcal{D}$. Полугруппа $\widehat{\mathcal{D}}$ содержит группу $\widehat{S}_{\triangle}$ матриц, соответствующих аффинным симметриям $S_{\triangle}$ треугольника $\triangle$. Если матрица $M$ принадлежит полугруппе $\widehat{\mathcal{D}} \subset \mathrm{GL}_{3}(\mathbb{Z})$, то по определению она допускает разложение в конечное произведение симметрий $\widehat{s} \in \widehat{S}_{\triangle}$ и элементарных возвратных матрии, $\widehat{\delta}_{*}^{k l} \in \widehat{\mathcal{D}}$, определенных в (3.14). Произвольную матрицу $M$ из полугруппы $\widehat{\mathcal{D}}$ также будем называть возвратной. Из определения $\mathcal{D}$ следует, что такая матрица $M$ будет унимодулярной.

Чтобы избежать длинных записей и сделать их более удобочитаемыми, воспользуемся следующими сокращениями:

$$
\widehat{s} \rightarrow s, \quad\left(\widehat{\delta}_{k}^{k l}\right)^{n} \rightarrow k l^{n}, \quad\left(\widehat{\delta}_{l}^{k l}\right)^{n} \rightarrow l k^{n}
$$

для $n=0,1,2, \ldots$. Например, разложение

$$
M=\widehat{s} \cdot \widehat{\delta}_{k_{1}}^{k_{1} l_{1}} \cdot\left(\widehat{\delta}_{l_{2}}^{k_{2} l_{2}}\right)^{3}
$$

примет сокращенный вид

$$
M=s \cdot k_{1} l_{1} \cdot l_{2} k_{2}^{3}
$$

Среди разложений матриц $M(a)$ в произведение симметрий $\widehat{s}$ и элементарных возвратных матриц $\widehat{\delta}_{*}^{k l}$ можно выделить две основные серии. В предложениях 4.1 и 4.2 представлены указанные разложения матриц $M(a)$ для $\triangle_{\mathrm{irr}}$-многочленов $f(x)$ вида $(4.1)$.

4.4. Серия $\boldsymbol{a}_{0}=\mathbf{- 1}$. В предложении 4.1 представлена основная серия разложений матриц $M(a)$, соответсвующая коэффициенту $a_{0}=-1$. Другая серия с коэффициентом $a_{0}=1$ содержится в предложении 4.2.

ПрЕДЛОЖЕНИЕ 4.1. Пусть $a_{0}=-1$. Тогда в обозначениях (4.5) матрици $М(a)$ из (4.3) имеют следующие разложения:

$$
\begin{aligned}
& M(a)=r_{1} \cdot \underline{01 \cdot 20} \cdot 02^{a_{1}-1} \cdot 01^{a_{2}-2} \quad \text { для } a_{2} \geqslant 2, \quad a_{1} \geqslant 1, \\
& M(a)=r_{1} \cdot \underline{21 \cdot 20} \cdot 02^{a_{1}-1} \quad \text { для } a_{2}=1, \quad a_{1} \geqslant 1, \\
& M(a)=r_{1} \cdot \underline{20} \cdot 02^{\left|a_{2}\right|+1} \cdot \underline{21} \cdot 02^{a_{1}-\left|a_{2}\right|-2} \quad \text { для } \quad a_{2} \leqslant 0, \quad a_{1} \geqslant\left|a_{2}\right|+2 .
\end{aligned}
$$

ЗАМЕчАНИЕ 4.1. В разложениях (4.6)-(4.8) подчеркиванием выделен остов или фрейм разложения, сохраняющийся при всех изменениям параметров $a_{1}, a_{2}$ в заданных пределах.

ДоКАЗАТЕЛЬСТВо получается прямыми вычислениями с использованием формул

$$
01^{a}=\left(\begin{array}{ccc}
1 & 0 & 0 \\
0 & 1 & 0 \\
-a & 0 & 1
\end{array}\right), \quad 02^{a}=\left(\begin{array}{ccc}
1 & 0 & 0 \\
0 & 1 & 0 \\
0 & -a & 1
\end{array}\right)
$$

4.5. Серия $\boldsymbol{a}_{0}=\mathbf{1}$. Для данного случая основная серия разложений матриц $M(a)$ аналогична основной серии разложений для $a_{0}=-1$.

ПреДлОЖЕНИЕ 4.2. Пусть $a_{0}=1$. Тогда для матрии, $M($ a) выполняются следующие разложения:

$$
\begin{aligned}
& M(a)=h_{0} \cdot \underline{20} \cdot 02^{a_{2}} \cdot \underline{21} \cdot 02^{\left|a_{1}\right|-a_{2}-2} \quad \text { для } \quad a_{2} \geqslant 1, \quad a_{1} \leqslant-a_{2}-3, \\
& M(a)=h_{0} \cdot \underline{01 \cdot 20} \cdot 02^{\left|a_{1}\right|-2} \cdot 01^{\left|a_{2}\right|-1} \quad \text { для } \quad a_{2} \leqslant-1, \quad a_{1} \leqslant-2 .
\end{aligned}
$$




\section{5. Неподвижные точки возвратных матриц}

5.1. Соответствие и специализации. Выписанные ранее разложения матрицы $M(a)$ в виде произведения симметрии $\widehat{s}$ и элементарных возвратных матриц $\widehat{\delta}_{*}^{k l}$ задают соответствие

$$
M(a) \rightarrow \delta_{s}
$$

где отображение $\delta_{s}=s \delta$ принадлежит расширенной полугруппе $\mathcal{D}$ и равно композиции симметрии $s$ и некотрого отображения $\delta$ из полугруппы $\mathcal{D}_{\delta} \subset \mathcal{D}$. Более того, соответствие $(5.1)$ определяет также и специализацию $\delta_{*}$ второго отображения: $\delta$ равно произведению элементарных возвратных отображений $\delta^{k l}$, а его специализация $\delta_{*}$ равна произведению специализаций $\delta_{*}^{k l}$, определяемых элементарными возвратными матрицами $\widehat{\delta}_{*}^{k l}$, содержащимися в правой части разложений матрицы $M(a)$.

\section{2. Неподвижные точки.}

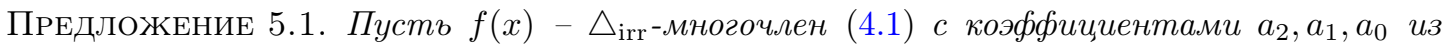
предложений 4.1 и 4.2 (см. (4.6)-(4.10)) и $\alpha$ - точка из области $\triangle^{\mathrm{int}}$, определенная в (4.2). Кроме того, пусть $M(a)$ - возвратная матрича (4.3) с параметром $a=\left(a_{2}, a_{1}, a_{0}\right) u \delta_{s}=$

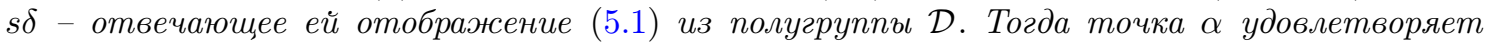
условиям

$$
\alpha \in \triangle\left(\delta_{s *}\right),
$$

где $\triangle\left(\delta_{s *}\right)$ - область определения специализации $\delta_{s *}=s \delta_{*}$ отображения $\delta_{s}$, задаваемой соответствием (5.1), и

$$
\delta_{s *} \alpha=\alpha,
$$

т.е. точка $\alpha$ допускается специализачией $\delta_{s *}$ и является неподвижной точкой отображения $\delta_{s}: \delta_{s} \alpha=\alpha$.

ДокАЗАТЕЛЬСТво. По условию точка $\alpha$ удовлетворяет $\triangle_{\mathrm{irr}}$-условию и по построению $(4.3)$ матрицы $M(a)$ отвечающий точке $\alpha$ вектор-столбец $\widehat{\alpha}$ является (4.4) собственным $M(a) \widehat{\alpha}=\lambda \widehat{\alpha}$ для матрицы $M(a)$ с собственным значением $\lambda=\theta \neq 0$. Поэтому можно применить вторую часть теоремы 5.1 [16], из которой вытекают оба утверждения (5.2) и (5.3).

Пусть $x=\left(x_{1}, x_{2}\right)$ - произвольная точка с координатами из $\mathbb{R}$. Обозначим через $F_{x}=$ $\mathbb{Q}\left(x_{1}, x_{2}\right)$ расширение, являющееся полем и состоящее из всех чисел вида $f\left(x_{1}, x_{2}\right) / g\left(x_{1}, x_{2}\right)$, где $f$ и $g$ - многочлены над $\mathbb{Q}$. Определим степень (над $\mathbb{Q}$ ) точки $x$ равенством

$$
\operatorname{deg}(x)=\operatorname{deg} F_{x},
$$

где $\operatorname{deg} F_{x}=\left[F_{x}: \mathbb{Q}\right]-$ степень поля $F_{x}$.

ТЕОРЕма 5.1 [1]. Пусть точка $x$ будет иррационалъной (3.18). Если при этом она является неподвижной точкой некоторого отображения $\delta$ из полугруппы $\mathcal{D}$, то ее степенъ

$$
\operatorname{deg}(x)=3
$$

\section{6. Дифференцирования и нормирования звезды}

6.1. Коммутативная диаграмма. Далее будем предполагать, что специализация $\delta_{*}$, задаваемая точкой $\alpha$, имеет вид

$$
\delta_{*}=\delta_{p} \cdots \delta_{2} \delta_{1}
$$

где $\delta_{i}=\delta_{*}^{k_{i} l_{i}}-$ специализации, определяемые соответствием (5.1). Заметим, что отображение (6.1) не содержит симметрии $s$ из группы $S_{\triangle}$. 
Рассмотрим следующую диаграмму:

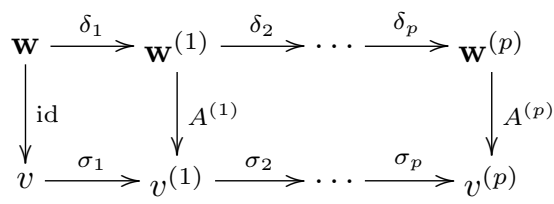

Здесь использовали такие обозначения:

- $\mathbf{w}=\mathbf{w}^{(0)}-$ начальная звезда с центром в точке $x=x^{(0)}=\alpha$;

- $\mathbf{w}^{(1)}=\delta_{1} \mathbf{w}^{(0)}-$ звезда с центром в $x^{(1)}=\delta_{1} x^{(0)}$, и т.д.;

- $\mathbf{w}^{(p)}=\delta_{p} \mathbf{w}^{(p-1)}-$ звезда с центром в $x^{(p)}=\delta_{p} x^{(p-1)}$.

Кратко цепочку преобразований из верхней строки диаграммы (6.2) можем записать в виде композиции

$$
\mathbf{w}^{(p)}=\delta_{p}\left(\ldots\left(\delta_{2}\left(\delta_{1} v\right)\right) \ldots\right)=\delta_{*} \mathbf{w}, \quad x^{(p)}=\delta_{p}\left(\ldots\left(\delta_{2}\left(\delta_{1} x\right)\right) \ldots\right)=\delta_{*} x
$$

из $p$ возвратных отображений $\delta_{*}$. Так определенные $\mathbf{w}^{(i)}$ для $i=1, \ldots, p$ будут, согласно определению (3.8), нормированными звездами. Нижняя строка диаграммы (6.2) содержит обычные (см. определение 1.1) или динамические звезды $v^{(i)}$ для $i=1, \ldots, p$, где

- $v=v^{(0)}=\mathbf{w}$;

- $v^{(1)}=v^{(0) \sigma_{1}}-$ производная звезда относительно дифференцирования $\sigma_{1}=\left\{k_{1}, l_{1}\right\}$, ассоциированного с отображением $\delta_{1}=\delta_{*}^{k_{1} l_{1}}$, и т.д.;

- $v^{(p)}=v^{(p-1) \sigma_{p}}-$ производная звезда относительно $\sigma_{p}=\left\{k_{p}, l_{p}\right\}$, ассоциированного с $\delta_{p}=\delta_{*}^{k_{p} l_{p}}$.

Следовательно, имеем представление

$$
v^{(p)}=\left(\left(v^{\sigma_{1}}\right)^{\sigma_{2}} \ldots\right)^{\sigma_{p}}=v^{\sigma}
$$

звезды $v^{(p)}$ через последовательность дифференцирований $\sigma=\sigma_{1} \sigma_{2} \ldots \sigma_{p}$, ассоциированную с отображением $\delta_{*}$ из (5.1).

Теперь опишем вертикальные стрелки из диаграммы (6.2). Первая стрелка обозначет тождественное отображение id, т.е. $v=\mathrm{id} \mathbf{w}=\mathbf{w}$. Далее, выпишем матрицы $A^{(p)}$ :

- $A^{(1)}=A_{*}^{k_{1} l_{1}}\left(x^{(0)}\right)$ - матрица $(3.5)$, зависящая от начальной точки $x=x^{(0)}$;

- $A^{(2)}=A_{*}^{k_{1} l_{1}}\left(x^{(0)}\right) A_{*}^{k_{2} l_{2}}\left(x^{(1)}\right)$ - матрица, уже зависящая от двух точек $x=x^{(0)}$ и $x^{(1)}$, и т.д.;

- последняя матрица

$$
A^{(p)}=A_{*}^{k_{1} l_{1}}\left(x^{(0)}\right) A_{*}^{k_{2} l_{2}}\left(x^{(1)}\right) \cdots A_{*}^{k_{p} l_{p}}\left(x^{(p-1)}\right)
$$

определяется всеми предыдущими точками $x^{(0)}, x^{(1)}, \ldots, x^{(p-1)}$.

Таким образом, по определению матрицы $A^{(i)}$ определяют аффинные изоморфизмы

$$
A^{(i)}: \mathbf{w}^{(i)} \vec{\sim} v^{(i)}
$$

звезд $\mathbf{w}^{(i)}$ и $v^{(i)}$ из верхней и нижней строк диаграммы $(6.2)$ для всех $i=1, \ldots, p$.

Лемма 6.1 [1]. Диаграмма (6.2) является коммутативной.

6.2. Периодические звезды. Из предложения 5.1 следует, что нормированная звезда $\mathbf{w}^{(p)}$ из диаграммы $(6.2)$ будет симметричной

$$
\mathbf{w}=\check{s} \mathbf{w}^{(p)}
$$


исходной звезде $\mathbf{w}=\mathbf{w}^{(0)}$ относительно преобразования $\breve{s}$ из группы $S_{\asymp}$ аффинных одно-

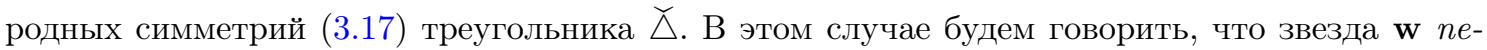
риодична относительно отображения $\delta_{s}$ из (5.1), а звезда $v=\mathbf{w}$ периодична относительно дифференцирования $\sigma$ из (6.4), при этом $p>0$ будет периодом звезды $v=\mathbf{w}$. Заметим, что однородные преобразования $\breve{s}$ из (3.17) действуют на векторы звезды w, а преобразования $s$ из группы $S_{\triangle}$ аффинных неоднородных симметрий $(3.15),(3.16)$ действуют на точки треугольника $\triangle$. В (6.7) звезда $\mathbf{w}$ - это совокупность трех векторов $\mathbf{w}_{0}, \mathbf{w}_{1}$ и $\mathbf{w}_{2}$.

6.3. Калибровочная матрица. Поскольку $v^{(p)}=A^{(p)} \mathbf{w}^{(p)}$ по формуле (6.6), то с помощью равенства (6.7) получаем

$$
v^{(p)}=A^{(p)} \mathbf{w}^{(p)}=A^{(p)} \breve{s}^{-1} \mathbf{w} .
$$

Поэтому, принимая во внимание равенство $\mathbf{w}=v$, можем записать

$$
v^{(p)}=\mathbf{A} v
$$

где матрица $\mathbf{A}=A^{(p)} \breve{s}^{-1}$ определяет аффинное однородное отображение звезд $\mathbf{A}: v \mapsto v^{(p)}$. Равенство (6.8) означает, что производная звезда $v^{(p)}$ из диаграммы (6.2) афббино изоморфна начальной звезде $v$. По этой причине $\mathbf{A}$ называется калибровочной матриией периодической звезды $v$. Далее мы хотим воспользоваться равенством (6.8) несколько раз. С этой целью рассмотрим бесконечную периодическую комбинированную последовательность $\xi=\left\{\xi_{0}, \xi_{1}, \ldots\right\}$ с периодом $p$, где

$$
\xi_{1}=\sigma_{1}, \quad \xi_{2}=\sigma_{2}, \quad \ldots, \quad \xi_{p}=\sigma_{p} \check{s}^{-1}, \quad \xi_{p+1}=\sigma_{1}, \quad \ldots
$$

С помощью последовательности $\xi$ определим для звезды $v$ по индукции звезды $v^{(i)}$ для $i=$ $0,1,2, \ldots$, полагая

$$
v^{(i)}=\left(v^{(i-1)}\right)^{\xi_{i}} \quad \text { для } n \geqslant 1,
$$

где $v^{(0)}=v$ и $v^{\prime \xi_{i}}=\check{s}\left(v^{\prime \sigma_{p}}\right)$ для $i=p, 2 p, 3 p, \ldots$. Чтобы не вводить новый термин, будем так определенные звезды $v^{(i)}$ продолжать называть производными для звезды $v$.

ТЕОРема 6.1 [1]. Пустъ звезда $v$ периодична относителъно дифферениирования $\sigma$ из (6.4) с периодом р, и пусть $\mathbf{A}$ - ее калибровочная матрица, определенаая в (6.8). Тогда для всех $i=0,1,2, \ldots$ справедлива формула

$$
v^{(i)}=\mathbf{A}^{a} v^{(b)},
$$

если $i=a t+b$, где $a=0,1,2, \ldots u b=0, \ldots, p-1$.

\section{7. Приближения на торе}

7.1. Генерации вкладывающихся разверток. По определению (2.18) и теореме 2.3 производные звезды $v^{(i)}$ из (6.9) вкладываются $v^{(i)} \stackrel{\text { em }}{\hookrightarrow} \mathbb{T}^{2}$ в тор $\mathbb{T}^{2}=\mathbb{R}^{2} / \mathbb{Z}^{2}$. По соглашению (2.16) это означает, что порождаемые ими ядра или шестиугольники $T^{(i)}=T\left(v^{(i)}\right)$ вкладываются в тор

$$
T^{(i)} \stackrel{\mathrm{em}}{\hookrightarrow} \mathbb{T}^{2}
$$

Из построения (6.9) производной звезды $v^{(i)}=\left\{v_{0}^{(i)}, v_{1}^{(i)}, v_{2}^{(i)}\right\}$ следует, что ее векторы перекладывания $v_{k}^{(i)}$ имеют вид

$$
v_{k}^{(i)} \equiv m_{k}^{(i)} \alpha^{-} \bmod \mathbb{Z}^{2}
$$


для $k=0,1,2$ с некоторыми коэффициентами $m_{k}^{(i)}=1,2,3, \ldots$, которые назовем порядками лучей $v_{k}^{(i)}$ звезды $v^{(i)}$. Здесь $\alpha^{-}=-\alpha \in \mathbb{R}^{2}$ - вектор сдвига $S=S_{\alpha^{-}}$тора $\mathbb{T}^{2}$ из (2.7) и порядки $m_{k}^{(i)}$ вычисляются по правилу (1.4). Сумму данных коэффициентов

$$
m^{(i)}=m_{0}^{(i)}+m_{1}^{(i)}+m_{2}^{(i)}
$$

назовем порядком производной звезды $v^{(i)}$. С ним свяжем конечные орбиты

$$
\operatorname{Orb}^{\prime}\left(0, m^{(i)}\right)=\left\{x_{j}=S^{j}(0) \equiv j \alpha^{-} \bmod \mathbb{Z}^{2}: j=1,2, \ldots, m^{(i)}-1\right\} .
$$

7.2. Спектральный радиус. Пусть $\lambda_{1}, \lambda_{2}$ - собственные значения невыродженной комплексной $2 \times 2$-матрицы $A$ и

$$
\varrho(A)=\max \left\{\left|\lambda_{1}\right|,\left|\lambda_{2}\right|\right\}
$$

- ее спектральный радиус. Рассмотрим наиболее интересный случай $\lambda_{1} \neq \lambda_{2}$. Такую матрицу $A$ можно представить в ввиде произведения $A=M J M^{-1}$ диагональной матрицы

$$
J=\left(\begin{array}{cc}
\lambda_{1} & 0 \\
0 & \lambda_{2}
\end{array}\right)
$$

и некоторой невырожденной комплексной матрицы $M$. Введем на $\mathbb{C}^{2} s$-метрикy $|x|_{s}=\left|x_{1}\right|+$ $\left|x_{2}\right|$, обладающую свойством

$$
|M x|_{s} \leqslant 2\|M\|_{\max } \cdot|x|_{s}
$$

где

$$
M=\left(\begin{array}{ll}
m_{11} & m_{12} \\
m_{21} & m_{22}
\end{array}\right)
$$

- произвольная комплексная матрица,

$$
M x=M\left(\begin{array}{l}
x_{1} \\
x_{2}
\end{array}\right), \quad\|M\|_{\max }=\max _{i, j}\left|m_{i j}\right|
$$

- $\operatorname{max-норма~матрицы~} M$. Используя разложение $A=M J M^{-1}$ и неравенство (7.4) имеем

$$
\begin{aligned}
|A x|_{s} & =\left|M\left(J M^{-1} x\right)\right|_{s} \leqslant 2\|M\|_{\max }\left|J\left(M^{-1} x\right)\right|_{s} \\
& \leqslant 4\|M\|_{\max }\|J\|_{\max }\left|M^{-1} x\right|_{s} \leqslant 8\|M\|_{\max }\left\|M^{-1}\right\|_{\max }\|J\|_{\max }|x|_{s} .
\end{aligned}
$$

Так как $\|J\|_{\max }=\max _{i}\left|\lambda_{i}\right|=\varrho(A)$, то приходим к следующему неравенству:

$$
|A x|_{s} \leqslant c_{s}(M) \varrho(A)|x|_{s}
$$

с константой $c_{s}(M)=8\|M\|_{\max }\left\|M^{-1}\right\|_{\max }$. Снова используя разложение $A=M J M^{-1}$ можем записать $A^{n}=M J^{n} M^{-1}$, откуда выводим $\varrho\left(J^{n}\right)=\varrho(A)^{n}$, поскольку $\varrho\left(J^{n}\right)=\varrho(J)^{n}=\varrho(A)^{n}$. Теперь, еще раз применяя схему $(7.5),(7.6)$ и равенство $\varrho\left(J^{n}\right)=\varrho(A)^{n}$, получаем общее неравенство

$$
\left|A^{n} x\right|_{s} \leqslant c_{s}(M) \varrho(A)^{n}|x|_{s}
$$

для любой степени $n=1,2,3, \ldots$. Неравенство (7.7) также переносится и на евклидову метрику $|x|=\left(\left|x_{1}\right|^{2}+\left|x_{2}\right|^{2}\right)^{1 / 2}$. Повторяя рассуждения (7.5)-(7.7) и применяя неравенства

$$
\frac{1}{\sqrt{2}}|x|_{s} \leqslant|x| \leqslant|x|_{s},
$$

можно получить аналог неравенства (7.7) для евклидовой метрики

$$
\left|A^{n} x\right| \leqslant c(M) \varrho(A)^{n}|x|
$$

для любой степени $n=1,2,3, \ldots$ с другой увеличенной константой $c(M)=\sqrt{2} c(M)_{s}$. 
7.3. Радиусы и площади производных шестиугольников. Применим доказанные формулы (7.7), (7.8) к оценке метрических характеристик производных шестиугольников $T^{(i)}=T\left(v^{(i)}\right)$ из (7.1). Как обычно, радиус множества $T \subset \mathbb{R}^{2}$ равен минимальному из радиусов окружностей, содержащих в себе указанное множество. В соответствии с метрикой $|x|_{s}$ и $|x|$ будем использовать два радиуса $r_{s}\left(T^{(i)}\right)$ и $r\left(T^{(i)}\right)$.

Лемма 7.1. Если шестиугольник $T(v)$ порождается звездой $v=\left\{v_{0}, v_{1}, v_{2}\right\}$, то его радиусы $r_{*}(T(v))$ вычисляется по формуле

$$
r_{*}(T(v))=\frac{1}{2} \max \left\{\left|v_{0}+v_{1}-v_{2}\right|_{*},\left|v_{0}-v_{1}+v_{2}\right|_{*},\left|-v_{0}+v_{1}+v_{2}\right|_{*}\right\},
$$

где $r_{*}(T(v))$ может бъть любъм из радиусов $r_{s}(T(v))$ или $r(T(v))$ в зависимости от въбранной метрики $|x|_{*}$, равной $|x|_{s}$ или $|x|$.

ДокАЗАТЕЛЬСтво. Из определения (2.6) следует, что шестиугольник $T(v)$ имеет вершины $v_{k}, v_{k^{\prime}}+v_{k^{\prime \prime}}$, где $k=0,1,2,0 \leqslant k^{\prime}<k^{\prime \prime} \leqslant 2$. Поэтому его центром будет

$$
c(T(v))=\frac{1}{2}\left(v_{0}+v_{1}+v_{2}\right) .
$$

Радиус же шестиугольника $r_{*}(T(v))$ равен максимальной длине векторов $w-c(T(v))$, где $w-$ вершины $T(v)$. Отсюда получаем (7.9).

Шестиугольник $T^{(i)}$ является разверткой тора $\mathbb{T}_{L^{(i)}}^{2}=\mathbb{R}^{2} / L^{(i)}$ для решетки $L^{(i)}=\mathbb{Z}\left[l_{1}^{(i)}, l_{2}^{(i)}\right]$ с базисом $l_{k}^{(i)}=v_{k}^{(i)}-v_{0}^{(i)}$ для $k=1,2$, где $v_{k}^{(i)}-$ лучи звезды $v^{(i)}$ из п. 7.1. Поэтому площадь $s\left(T^{(i)}\right)$ шестиугольника $T^{(i)}$ равна

$$
s\left(T^{(i)}\right)=\left|\operatorname{det}\left(\begin{array}{ll}
l_{11}^{(i)} & l_{12}^{(i)} \\
l_{21}^{(i)} & l_{22}^{(i)}
\end{array}\right)\right|
$$

- площади фундаментальной области решетки $L^{(i)}$, где $l_{k l}^{(i)}$ - координаты базисных векторов $l_{k}^{(i)}$ для $k=1,2$.

Лемма 7.2. 1. Если собственнъе значения $\lambda_{1} \neq \lambda_{2}$ калибровочной матрицъ $\mathbf{A}$ звезды $v$ из (6.8), то для радиусов $r_{*}\left(T^{(i)}\right)=r_{s}\left(T^{(i)}\right)$ или $r\left(T^{(i)}\right)$ шестиугольника $T^{(i)}$ выполняется оценка

$$
r_{*}\left(T^{(i)}\right) \leqslant c_{*}(M) \varrho(\mathbf{A})^{a} r_{*}\left(T^{(b)}\right)
$$

для $i=a p+b$, где $a=0,1,2, \ldots u b=0, \ldots, p-1$. Здесъ константы $c_{*}(M)$ равны $c_{s}(M)$ из (7.6) или $c(M)=\sqrt{2} c(M)_{s}$ и $\varrho(A)$ - спектралъный радиус матрицы $\mathbf{A}$.

2. Площадь $s\left(T^{(i)}\right)$ шестиугольника $T^{(i)}$ находится по формуле

$$
s\left(T^{(i)}\right)=|\operatorname{det} \mathbf{A}|^{a} s\left(T^{(b)}\right),
$$

где $\operatorname{det} \mathbf{A}$ обозначает определитель матрищы $\mathbf{A}$, удовлетворяющий неравенствам $0<$ $|\operatorname{det} \mathbf{A}|<1$, а площади s( $\left.T^{(b)}\right)$ находятся по формуле (7.10).

ДокАЗАтЕЛьство. По теореме 6.1 звезды $v^{(i)}$ и $v^{(b)}$ связаны равенством $v^{(i)}=\mathbf{A}^{a} v^{(b)}$ и, значит, порождаемые ими шестиугольники $T^{(i)}=T\left(v^{(i)}\right)$ и $T^{(b)}=T\left(v^{(b)}\right)$ аффинно подобны $T\left(v^{(i)}\right)=\mathbf{A}^{a} T\left(v^{(b)}\right)$. Отсюда, и из (7.7) и (7.8) следует неравенство (7.11). Формула (7.12) вытекает из подобия и равенства (7.10). Для доказательства же неравенств $0<|\operatorname{det} \mathbf{A}|<1$ достаточно заметить, что по построению (6.5), (6.8) калибровочная матрица А состоит из сомножителей $A_{*}^{k l}$, имеющих определители $0<\left|\operatorname{det} A_{*}^{k l}\right|<1$ согласно (3.5). 
7.4. Порядковые матрицы. Составим из порядков лучей звезды $v$ (см. п. 7.1) матрицу-столбец

$$
\mathbf{m}=\mathbf{m}(v)=\left(\begin{array}{l}
m_{0} \\
m_{1} \\
m_{2}
\end{array}\right)
$$

и определим матрицы, соответствующие симметриям (3.15), (3.16) базисного реугольника:

$$
\begin{array}{lll}
R_{0}=\left(\begin{array}{lll}
1 & 0 & 0 \\
0 & 1 & 0 \\
0 & 0 & 1
\end{array}\right), & R_{1}=\left(\begin{array}{lll}
0 & 0 & 1 \\
1 & 0 & 0 \\
0 & 1 & 0
\end{array}\right), & R_{2}=\left(\begin{array}{lll}
0 & 1 & 0 \\
0 & 0 & 1 \\
1 & 0 & 0
\end{array}\right), \\
H_{0}=\left(\begin{array}{lll}
1 & 0 & 0 \\
0 & 0 & 1 \\
0 & 1 & 0
\end{array}\right), & H_{1}=\left(\begin{array}{lll}
0 & 0 & 1 \\
0 & 1 & 0 \\
1 & 0 & 0
\end{array}\right), & H_{2}=\left(\begin{array}{lll}
0 & 1 & 0 \\
1 & 0 & 0 \\
0 & 0 & 1
\end{array}\right) ;
\end{array}
$$

и - дробно-линейным преобразованиям или дифференцированиям (3.14):

$$
\begin{array}{rlrl}
D_{1}^{12} & =\left(\begin{array}{lll}
1 & 0 & 0 \\
0 & 1 & 0 \\
0 & 1 & 1
\end{array}\right), & D_{2}^{12}=\left(\begin{array}{lll}
1 & 0 & 0 \\
0 & 1 & 1 \\
0 & 0 & 1
\end{array}\right), \\
D_{0}^{01}=\left(\begin{array}{lll}
1 & 0 & 0 \\
1 & 1 & 0 \\
0 & 0 & 1
\end{array}\right), & D_{1}^{01}=\left(\begin{array}{lll}
1 & 1 & 0 \\
0 & 1 & 0 \\
0 & 0 & 1
\end{array}\right), \\
D_{0}^{02}=\left(\begin{array}{lll}
1 & 0 & 0 \\
0 & 1 & 0 \\
1 & 0 & 1
\end{array}\right), & D_{2}^{02}=\left(\begin{array}{lll}
1 & 0 & 1 \\
0 & 1 & 0 \\
0 & 0 & 1
\end{array}\right),
\end{array}
$$

Матрицы (7.13) и (7.14) принадлежат группе унимодулярных матриц $\mathrm{GL}_{3}(\mathbb{Z})$.

Данные матрицы позволяют вычислять порядки лучей $m_{0}^{\sigma}, m_{1}^{\sigma}, m_{2}^{\sigma}$ преобразованной звезды $v^{\sigma}=\left(v_{0}^{\sigma}, v_{1}^{\sigma}, v_{2}^{\sigma}\right)$ :

$$
\mathbf{m}^{\sigma}=\mathbf{m}\left(v^{\sigma}\right)=M^{\sigma}(v) \mathbf{m} .
$$

Здесь согласно определению производной (1.4) имеем

$$
M^{\sigma}(v)=D_{*}^{k_{1} k_{2}},
$$

если $\sigma=\left\{k_{1}, k_{2}\right\}$ - дифференцирование. Здесь в качестве специализации $*$ выбирается $k_{1}$ или $k_{2}$ в зависимости от того, какое из условий (1.4) выполняется;

$$
M^{\sigma}(v)=M^{\sigma}=S_{k}
$$

в случае преобразования симметрии $\sigma=s$ звезды $v$, где $S_{k}=R_{k}$ или $H_{k}-$ соответствующая матрица из (7.13).

ЛЕмма 7.3 [1]. Пусть $v-$ периодическая звезда $v^{(p)}=\mathbf{A} v$ периода $p>0$ с калибровочной матрицей $\mathbf{A}=A^{(p) \breve{s}^{-1}}$ из (6.8), и пусть ее производные звезды $v^{(i)}$ для $i=0,1,2, \ldots$ определены по формуле (6.9). Тогда если $i=a p+b$, где $a=0,1,2, \ldots u b=0, \ldots, p-1$, mо

$$
\mathbf{m}^{(i)}=\mathbf{m}\left(v^{(i)}\right)=\mathbf{M}^{a} \mathbf{m}^{(b)} .
$$

Здесь $\mathbf{M}=S M^{(p)}$ с матрищей $S$ из (7.13), соответствующей обратной симметрии для $\check{s}, u$

$$
M^{(p)}=D_{*}^{k_{p} l_{p}}\left(v^{(p-1)}\right) \cdots D_{*}^{k_{2} l_{2}}\left(v^{(1)}\right) D_{*}^{k_{1} l_{1}}\left(v^{(0)}\right),
$$

где у порядковъх матрии $D_{*}^{k_{j} l_{j}}\left(v^{(j-1)}\right)=D_{*}^{k_{j} l_{j}}$ из (7.15) специализации * определяются производной звездой $v^{(j-1)}$.

Назовем М порядковой матрицей периодической звезды $v$, отвечающей калибровочной матрице А. 
7.5. Рекуррентные последовательности. Рассмотрим векторную рекуррентную последовательность

$$
\mathbf{f}^{i+1}=M \mathbf{f}^{i}
$$

для $i=0,1,2, \ldots$. Здесь $M-3 \times 3$-матрица, $\mathbf{f}^{i}=\left(\begin{array}{lll}f_{0}^{i} & f_{1}^{i} & f_{2}^{i}\end{array}\right)^{T}$ и $\mathbf{f}^{0}$ - некоторый фиксированный столбец. Из определения (7.17) следует формула

$$
\mathbf{f}^{i}=M^{i} \mathbf{f}^{0}
$$

Наша цель - найти рекуррентную зависимость для скалярной последовательности

$$
f^{i}=\mathbf{1} \cdot \mathbf{f}^{i}
$$

где $\mathbf{1}=\left(\begin{array}{lll}1 & 1 & 1\end{array}\right)$ - строка, т.е. $f^{i}=f_{0}^{i}+f_{1}^{i}+f_{2}^{i}$ определяются как суммы элементов столбов $\mathbf{f}^{i}$.

Для матрицы $M$ запишем ее характеристический многочлен в виде

$$
c h_{M}(x)=\operatorname{det}(x E-M)=x^{3}-b_{2} x^{2}-b_{1} x-b_{0} .
$$

По теореме Гамильтона-Кэли $M$ удовлетворяет матричному уравнению $M^{3}-b_{2} M^{2}-b_{1} M-$ $b_{0} E=0$. Перепишем его в виде равенства $M^{3}=b_{2} M^{2}+b_{1} M+b_{0} E$. Умножая его на столбец $\mathbf{f}^{i}$, получаем

$$
M^{3} \mathbf{f}^{i}=b_{2} M^{2} \mathbf{f}^{i}+b_{1} M \mathbf{f}^{i}+b_{0} \mathbf{f}^{i} .
$$

Теперь, применяя к равенству (7.21) формулу (7.18), приходим к новой рекуррентной формуле

$$
\mathbf{f}^{i+3}=b_{2} \mathbf{f}^{i+2}+b_{1} \mathbf{f}^{i+1}+b_{0} \mathbf{f}^{i}
$$

для столбцов $\mathbf{f}^{i}$. После этого умножим обе части равенства $(7.22)$ на строку $\mathbf{1}=(111)$ и воспользуемся равенством (7.19). Таким образом, приходим к следующему результату.

ПрЕДЛОЖЕНИЕ 7.1. Если числа $f^{i}$ для $i=0,1,2, \ldots$ определенъ равенством (7.19), то они удовлетворяют рекуррентному соотношению

$$
f^{i+3}=b_{2} f^{i+2}+b_{1} f^{i+1}+b_{0} f^{i}
$$

где $b_{2}, b_{1}, b_{0}$ - коэффичиенты характеристического многочлена (7.20) и начальнье условия

$$
f^{2}=\mathbf{1} \cdot M^{2} \mathbf{f}^{0}, \quad f^{1}=\mathbf{1} \cdot M \mathbf{f}^{0}, \quad f^{0}=\mathbf{1} \cdot \mathbf{f}^{0}
$$

задаются фиксированным столбиом $\mathbf{f}^{0}$ из (7.17).

7.6. Основная теорема (общий случай). Важность последовательности определенных ранее в (7.1) производных шестиугольников $T^{(i)}=T\left(v^{(i)}\right)$ - ядер индуцированных разбиений из п. 2.5 - для $i=0,1,2, \ldots$ объясняется тем, что через их геометрию характеризуются аппроксимационные свойства точек из бесконечной орбиты

$$
\operatorname{Orb}_{\alpha^{-}}(0)=\left\{x_{j}=S^{j}(0) \equiv j \alpha^{-} \bmod \mathbb{Z}^{2}: j=0,1,2, \ldots\right\},
$$

порождаемой сдвигом $S=S_{\alpha^{-}}$тора $\mathbb{T}^{2}=\mathbb{R}^{2} / \mathbb{Z}^{2}$ из (2.7). Ограниченные шестиугольниками $T^{(i)}$ области на торе $\mathbb{T}^{2}$ выделяют из орбиты (7.25) некоторую подпоследовательность точек $\left\{x_{j^{\prime}}\right\}_{j^{\prime}=1}^{\infty}$, наилучшим образом приближающихся к $0 \in \mathbb{T}^{2}$.

Теорема 7.1. В условиях предложений 4.1 u 4.2 пусть $v$ - периодическая звезда периода $p>0$ с калибровочной матрицей $\mathbf{A}$ из (6.8), и пусть ее производные звездь $v^{(i)}$ для $i=$ $0,1,2, \ldots$ определены по формуле (6.9). Тогда справедливы следующие утверждения. 
1) Ни одна из точек $x_{j} \equiv j \alpha^{-} \bmod \mathbb{Z}^{2}$ орбить (7.25) не попадает

$$
x_{j} \notin T^{(i)} \quad \text { для } \quad j=1,2, \ldots, m^{(i)}-1
$$

в шестиугольник $T^{(i)}$ из (7.1), где $m^{(i)}$ - порядок $(7.2)$ звездь $v^{(i)}$, равный сумме коэфбициентов матриць-столбеца $\mathbf{m}^{(i)}$ из (7.16). Первой попавшей в область $T^{(i)}$ является точка

$$
x_{j} \in T^{(i)} \quad \partial л я \quad j=m^{(i)} .
$$

2) Для радиуса $r_{*}\left(T^{(i)}\right)=r_{s}\left(T^{(i)}\right)$ или $r\left(T^{(i)}\right)$ шестиугольника $T^{(i)}$ с номером $i=a p+b$, где $a=0,1,2, \ldots$ и $b=0, \ldots, p-1$, выполняется следующее неравенство

$$
r_{*}\left(T^{(i)}\right) \leqslant c_{*}(M) \varrho(\mathbf{A})^{a} r_{*}\left(T^{(b)}\right),
$$

если собственные значения $\lambda_{1} \neq \lambda_{2}$ у калибровочной матрицы $\mathbf{A}$ звездъ $v$. Для начальных номеров b радиусы $r_{*}\left(T^{(b)}\right)$ в (7.28) вычисляются по бормуле (7.9). Здесь $\varrho(\mathbf{A})$ обозначает спектральный радиус калибровочной матричъ $\mathbf{A}$, и константы $c_{*}(M)$ определены в (7.7), (7.8).

3) Площадь $s\left(T^{(i)}\right)$ шестиугольника $T^{(i)}$ находится по формуле

$$
s\left(T^{(i)}\right)=|\operatorname{det} \mathbf{A}|^{a} s\left(T^{(b)}\right),
$$

где определитель $\operatorname{det} \mathbf{A}$ удовлетворяет неравенствам $0<|\operatorname{det} \mathbf{A}|<1$ и площади $s\left(T^{(b)}\right)$ для начальных номеров $b=0, \ldots, p-1$ вычисляются по формуле (7.10).

ДокАзАтельство. Утверждение 1) следует из теоремы 4.1 [15], а утверждения 2) и 3) доказаны в лемме 7.2.

\section{8. Двумерная ядерная аппроксимация}

8.1. Возвратные матрицы для кубических единиц и рекуррентные последовательности. Перепишем звезды $v^{(i)}=\left(v_{0}^{(i)}, v_{1}^{(i)}, v_{2}^{(i)}\right)$ из (6.9) в виде столбцов $v^{(i)}=$ $\left(\begin{array}{lll}v_{0}^{(i)} & v_{1}^{(i)} & v_{2}^{(i)}\end{array}\right)^{T}$. По определению имеем

$$
v^{(0)}=\left(\begin{array}{c}
v_{0}^{(0)} \\
v_{1}^{(0)} \\
v_{2}^{(0)}
\end{array}\right)=\left(\begin{array}{l}
v_{0} \\
v_{1} \\
v_{2}
\end{array}\right)=\left(\begin{array}{c}
-\alpha \\
-\alpha+e_{1} \\
-\alpha+e_{2}
\end{array}\right)
$$

или, по-другому,

$$
v^{(0)}=v=-\alpha E_{0}+e_{1} E_{1}+e_{2} E_{2},
$$

где

$$
E_{0}=\left(\begin{array}{l}
1 \\
1 \\
1
\end{array}\right), \quad E_{1}=\left(\begin{array}{l}
0 \\
1 \\
0
\end{array}\right), \quad E_{2}=\left(\begin{array}{l}
0 \\
0 \\
1
\end{array}\right) .
$$

При этом $\alpha, e_{1}, e_{2}$ в (8.2) рассматриваются как коэффициенты.

Пусть $i=a p+b$ с фиксированным $0 \leqslant b<p$. Определим сдвиги $\vec{j} \equiv j+b \bmod p$ и число $\vec{j}$ выбираем из интервала $[1, \ldots, p]$; для порядковой матрицы $\mathbf{M}=D_{p} \cdots D_{1}$ из $(7.16)$ с множителями $D_{j}=D_{*}^{k_{j} l_{j}}\left(v^{(j-1)}\right)$ и $D_{p}=S D_{*}^{k_{p} l_{p}}\left(v^{(p-1)}\right)$ соответственно для $j<p$ и $j=p$ полагаем $\overrightarrow{\mathbf{M}}=\vec{D}_{p} \cdots \vec{D}_{1}$, где $\vec{D}_{j}=\vec{D}_{j}$. Тогда в этих обозначениях из диаграммы (6.2) будет следовать формула

$$
v^{(i)}=\overrightarrow{\mathbf{M}}^{a} v^{(b)},
$$


где

$$
v^{(b)}=-\alpha E_{0}^{(b)}+e_{1} E_{1}^{(b)}+e_{2} E_{2}^{(b)},
$$

а столбцы $E_{i}^{(b)}$ вычисляются по формуле $E_{i}^{(b)}=D_{*}^{k_{b} l_{b}} \cdots D_{*}^{k_{1} l_{1}} E_{i}^{(0)}$ для $0 \leqslant b<p$. Здесь полагаем $E_{i}^{(0)}=E_{i}$ и $D_{*}^{k_{j} l_{j}}$ - порядковые матрицы из леммы 7.3. Тогда из (8.3) и (8.4) получаем

$$
v^{(i)}=-\alpha \overrightarrow{\mathbf{M}}^{a} E_{0}^{(b)}+e_{1} \overrightarrow{\mathbf{M}}^{a} E_{1}^{(b)}+e_{2} \overrightarrow{\mathbf{M}}^{a} E_{2}^{(b)} .
$$

Вводя следующие обозначения:

$$
\mathbf{Q}^{a}=\overrightarrow{\mathbf{M}}^{a} E_{0}^{(b)}, \quad \mathbf{R}_{1}^{a}=\overrightarrow{\mathbf{M}}^{a} E_{1}^{(b)}, \quad \mathbf{R}_{2}^{a}=\overrightarrow{\mathbf{M}}^{a} E_{2}^{(b)},
$$

перепишем равенство (8.5) в виде

$$
v^{(i)}=-\alpha \mathbf{Q}^{a}+e_{1} \mathbf{R}_{1}^{a}+e_{2} \mathbf{R}_{2}^{a} .
$$

Пусть $\mathbf{1}=\left(\begin{array}{lll}1 & 1 & 1\end{array}\right)$ - строка из (7.19). Тогда по теореме 7.1

$$
v_{\min }^{(i)}=\mathbf{1} \cdot v^{(i)}=v_{0}^{(i)}+v_{1}^{(i)}+v_{2}^{(i)}
$$

будет минимальным вектором (7.27), который в силу (8.7) можно записать в виде линейной комбинации

$$
v_{\min }^{(i)}=-\alpha Q^{a}+e_{1} R_{1}^{a}+e_{2} R_{2}^{a}
$$

с коэффициентами $Q^{a}=\mathbf{1} \cdot \mathbf{Q}^{a}, R_{1}^{a}=\mathbf{1} \cdot \mathbf{R}_{1}^{a}, R_{2}^{a}=\mathbf{1} \cdot \mathbf{R}_{2}^{a}$.

Из (8.6), (8.9) и предложения 7.1 следует, что $Q^{a}, R_{1}^{a}, R_{2}^{a}$ являются рекуррентными последовательностями от параметра $a$ (напомним, что параметр $b$ фиксирован):

$$
Q^{a+3}=b_{2} Q^{a+2}+b_{1} Q^{a+1}+b_{0} Q^{a}, \quad R_{k}^{a+3}=b_{2} R_{k}^{a+2}+b_{1} R_{k}^{a+1}+b_{0} R_{k}^{a}
$$

для $k=1,2$. Здесь $b_{2}, b_{1}, b_{0}$ - коэффициенты характеристического многочлена $c h \overrightarrow{\mathrm{M}}(x)=$ $c h_{\mathbf{M}}(x)$ порядковой матрицы $\mathbf{M}$, определенного в (7.20). Для последовательностей $Q^{a}$ и $R_{k}^{a}$ имеем следующие начальные условия:

$$
\begin{array}{lll}
Q^{0}=\mathbf{1} \cdot \mathbf{Q}^{0}=\mathbf{1} \cdot E_{0}^{(b)}, & Q^{1}=\mathbf{1} \cdot \mathbf{Q}^{1}=\mathbf{1} \cdot \overrightarrow{\mathbf{M}} E_{0}^{(b)}, & Q^{2}=\mathbf{1} \cdot \mathbf{Q}^{2}=\mathbf{1} \cdot \overrightarrow{\mathbf{M}}^{2} E_{0}^{(b)} \\
R_{k}^{0}=\mathbf{1} \cdot \mathbf{R}_{k}^{0}=\mathbf{1} \cdot E_{k}^{(b)}, & R_{k}^{1}=\mathbf{1} \cdot \mathbf{R}_{k}^{1}=\mathbf{1} \cdot \overrightarrow{\mathbf{M}} E_{k}^{(b)}, & R_{2}^{k}=\mathbf{1} \cdot \mathbf{R}_{k}^{2}=\mathbf{1} \cdot \overrightarrow{\mathbf{M}}^{2} E_{k}^{(b)}
\end{array}
$$

для $k=1,2$.

8.2. Основная теорема для кубических единиц. Общая теорема о ядерной аппроксимации 7.1 в применении к кубическим единицам допускает следующую конкретизацию.

Теорема 8.1. 1. Для $i=a p+b$ и любого $0 \leqslant b<p$ вектор

$$
v_{\min }^{(i)}=\left(-Q^{a} \alpha_{1}+R_{1}^{a},-Q^{a} \alpha_{2}+R_{2}^{a}\right)
$$

обладает минимальным свойством

$$
v_{\min }^{(i)} \in T^{(i)} \quad \text { сминимальным иельм } Q^{a} \geqslant 1 .
$$

Это означает, что ни одна из точек орбить $x_{j} \equiv-j \alpha \bmod \mathbb{Z}^{2}$ не попадает

$$
x_{j} \notin T^{(i)} \quad \text { для всех } 1 \leqslant j<Q^{a}
$$

в ядро $T^{(i)}$ - шестиугольник из (7.1). 
2. Площадь $s\left(T^{(i)}\right)$ ядра $T^{(i)}$ находится по формуле

$$
s\left(T^{(i)}\right)=|\operatorname{det} \mathbf{A}|^{a} s\left(T^{(b)}\right),
$$

где $\operatorname{det} \mathbf{A}$ обозначает определитель матрищы $\mathbf{A}$, удовлетворяющий неравенствам $0<$ $|\operatorname{det} \mathbf{A}|<1$, площади $s\left(T^{(b)}\right)$ для начальных номером $b=0, \ldots, p-1$ вычисляются по формуле (7.10).

3. Если у калибровочной матрищы $\mathbf{A}$ собственные значения $\lambda_{1} \neq \lambda_{2}$, то

$$
\left|Q^{a} \alpha_{1}-R_{1}^{a}\right|+\left|Q^{a} \alpha_{2}-R_{2}^{a}\right| \leqslant c_{s}^{(b)} \varrho(\mathbf{A})^{a},
$$

где $c_{s}^{(b)}=c_{s}(\vec{M}) r_{s}\left(v_{\min }^{(b)}\right)$.

4. Коэффичиенты $Q^{a}, R_{1}^{a}, R_{2}^{a}$ из (8.12) образуют рекуррентнъе последовательности от параметра $a=0,1,2, \ldots$ с уравнением (8.10) и начальными условиями (8.11).

5. Если $b=0$, то начальные условия рекуррентных последовательностей $Q^{a}, R_{1}^{a}, R_{2}^{a}$ принимают вид

$$
\begin{aligned}
Q^{0}=3, & Q^{1}=\sum_{k, l} m_{k l}, & Q^{2}=\sum_{k, l} m_{k l}^{2}, \\
R_{1}^{0}=1, & R_{1}^{1}=\sum_{k} m_{k 2}, & R_{1}^{2}=\sum_{k} m_{k 2}^{2}, \\
R_{2}^{0}=1, & R_{2}^{1}=\sum_{k} m_{k 3}, & R_{2}^{2}=\sum_{k} m_{k 3}^{2},
\end{aligned}
$$

где $\mathbf{M}=\left(m_{k l}\right)_{3 \times 3}-$ порядковая матрица из (7.16) и $\mathbf{M}^{2}=\left(m_{k l}^{2}\right)_{3 \times 3}-$ ее квадрат; длина же $r_{s}\left(v_{\min }^{(0)}\right)$ минимального вектора $(8.8)$, входящего в константу $c_{s}^{(0)}=c_{s}(\vec{M}) r_{s}\left(v_{\min }^{(0)}\right)$ из неравенств (8.16), равна

$$
r_{s}\left(v_{\min }^{(0)}\right)=\left|3 \alpha_{1}-1\right|+\left|3 \alpha_{2}-1\right|
$$

ДокАЗАтельство. 1. Формула (8.12) для минимального вектора $v_{\min }^{(i)}$ вытекает из равенства (8.9), его свойство минимальности (8.13), (8.14) - из теоремы 7.1.

2. Формула (8.15) для площади шестиугольника $s\left(T^{(i)}\right)$ была ранее доказана в лемме 7.2.

3. В левых частях неравенств (8.16) записана длина для минимального вектора $v_{\min }^{(i)}$ из (8.12) в $s$-метрике $|x|_{s}=\left|x_{1}\right|+\left|x_{2}\right|$. Поэтому указанные неравенства получаются как следствие из неравенств $(7.28)$, в которых радиусы $r_{*}\left(T^{(i)}\right)$ и $r_{*}\left(T^{(b)}\right)$ заменены длинами минимальных векторов $r_{s}\left(v_{\text {min }}^{(i)}\right)$ и $r_{s}\left(v_{\min }^{(b)}\right)$.

4. Рекуррентность коэффициентов $Q^{a}, R_{1}^{a}, R_{2}^{a}$ из (8.12) была ранее доказана в (8.10) и (8.11).

5. Явные начальные условия (8.17) для рекуррентных последовательностей $Q^{a}, R_{1}^{a}, R_{2}^{a}$ в случае $b=0$ также вычисляются по формулам (8.11). По определению (8.8) начальный минимальный вектор $v_{\min }^{(0)}=v_{0}^{(0)}+v_{1}^{(0)}+v_{2}^{(0)}=v_{0}+v_{1}+v_{2}$ равен сумме лучей исходной звезды $v^{(0)}=v=\left\{v_{0}, v_{1}, v_{2}\right\}$. Поскольку по определению $v_{0}=-\alpha, v_{1}=e_{1}-\alpha, v_{2}=e_{2}-\alpha$, то $v_{\min }^{(0)}=\left(1-3 \alpha_{1}, 1-3 \alpha_{2}\right)$, откуда вытекает равенство (8.18).

\section{9. Числовые примеры}

9.1. Кубическая единица Рози. В качестве (4.1) выберем многочлен

$$
f(x)=x^{3}+x^{2}+x-1,
$$


неприводимый над $\mathbb{Q}$. Он имеет вещественный корень $\theta=0.54 \ldots$ Поскольку $\alpha=\left(\alpha_{1}, \alpha_{2}\right) \in$

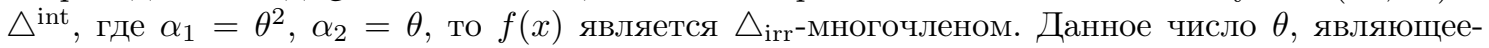
ся кубической единицей, ранее появлялось при построении двумерных квазипериодических разбиений Рози [17], [14].

Согласно (4.3) набору коэффициентов $a=\left(a_{2}, a_{1}, a_{0}\right)=(1,1,-1)$ многочлена (9.1) отвечает унимодулярная матрица

$$
M(a)=\left(\begin{array}{ccc}
-1 & -1 & 1 \\
1 & 0 & 0 \\
0 & 1 & 0
\end{array}\right)
$$

с определителем $\operatorname{det} M(a)=1$. Так как коэффициенты $a=\left(a_{2}, a_{1}, a_{0}\right)$ удовлетворяют условиям $a_{0}=-1, a_{2}=1$ и $a_{1} \geqslant 1$ из предложения 4.1, то в силу (4.7) матрица $M(a)$ имеет разложение $M(a)=r_{1} \cdot \underline{21 \cdot 20}$, которое, вспоминая соглашение $(4.5)$, можем записать в развернутом виде через симметрию треугольника и элементарные возвратные отображения

$$
M(a)=\left(\begin{array}{ccc}
-1 & -1 & 1 \\
1 & 0 & 0 \\
0 & 1 & 0
\end{array}\right)=\widehat{r}_{1} \cdot \widehat{\delta}_{2}^{12} \cdot \widehat{\delta}_{2}^{02}
$$

Поэтому по лемме 7.3 порядковая матрица $\mathbf{M}$, определенная в (7.16), будет равна

$$
\mathbf{M}=R_{1} \cdot D_{2}^{12} \cdot D_{2}^{02}=\left(\begin{array}{ccc}
0 & 0 & 1 \\
1 & 0 & 1 \\
0 & 1 & 1
\end{array}\right)
$$

Для матрицы $\mathbf{M}$ найдем ее характеристический многочлен $c h_{\mathbf{M}}(x)=x^{3}-b_{2} x^{2}-b_{1} x-b_{0}$ из (7.20). В силу (9.4) будем иметь

$$
\operatorname{ch}_{\mathbf{M}}(x)=\operatorname{det}\left(\begin{array}{ccc}
x & 0 & -1 \\
-1 & x & -1 \\
0 & -1 & x-1
\end{array}\right)=x^{3}-x^{2}-x-1
$$

и, значит, коэффициенты у многочлена $c h_{\mathbf{M}}(x)$ соответственно равны $b_{2}=1, b_{1}=1, b_{0}=1$.

Фиксируем параметр $b=0$. Тогда из теоремы 8.1 следует, что $Q^{a}, R_{1}^{a}, R_{2}^{a}$ являются рекуррентными последовательностями с одним и тем же уравнением

$$
Q^{a+3}=Q^{a+2}+Q^{a+1}+Q^{a}, \quad R_{k}^{a+3}=R_{k}^{a+2}+R_{k}^{a+1}+R_{k}^{a}
$$

для $k=1,2$. Последовательности (9.6) называются рекуррентными последователъностями Трибоначчи.

Принимая во внимание формулу (9.4) для порядковой матрицы $\mathbf{M}$ и затем вычисляя ее квадрат

$$
\mathbf{M}^{2}=\left(\begin{array}{lll}
0 & 1 & 1 \\
0 & 1 & 2 \\
1 & 1 & 2
\end{array}\right)
$$

по формулам (8.17) находим для рекуррентных последовательностей (9.6) начальные условия

$$
\begin{array}{lll}
Q^{0}=3, & Q^{1}=\sum_{k, l} m_{k l}=5, & Q^{2}=\sum_{k, l} m_{k l}^{2}=9, \\
R_{1}^{0}=1, & R_{1}^{1}=\sum_{k} m_{k 2}=1, & R_{1}^{2}=\sum_{k} m_{k 2}^{2}=3, \\
R_{2}^{0}=1, & R_{2}^{1}=\sum_{k} m_{k 3}=3, & R_{2}^{2}=\sum_{k} m_{k 3}^{2}=5 .
\end{array}
$$


Последовательности (9.6) вместе с начальными условиями (9.7) составляют единую рекуррентную последовательность

$$
R_{1}^{a}, R_{2}^{a}, Q^{a}: \quad 1,1,3,5,9,17,31,57,105, \ldots
$$

Здесь последовательность $R_{1}^{a}$ начинается с первого элемента из $(9.8), R_{2}^{a}-$ со второго, а $Q^{a}-$ с третьего.

Калибровочная матрица $\mathbf{A}=A^{(p)} \breve{r}_{2}$ из (6.8) с симметрией поворота $\breve{r}_{2}=\breve{r}_{1}^{-1}$ имеет два комплексно сопряженных собственных значения $\lambda_{1} \neq \lambda_{2}=\bar{\lambda}_{1}$. Поэтому можно применить неравенство (8.16) из теоремы 7.1. После вычислений приходим к следующей совместной аппроксимации с экспоненциальной оценкой для вещественного корня $\theta: \theta^{3}+\theta^{2}+\theta-1=0$, и его квадрата $\theta^{2}$.

Если $\theta$ - вещественный корень многочлена (9.1), то выполняется следующее неравенство

$$
\left|Q^{a} \theta^{2}-R_{1}^{a}\right|+\left|Q^{a} \theta-R_{2}^{a}\right| \leqslant c_{s}^{(0)} \varrho(\mathbf{A})^{a}
$$

для всех $a=0,1,2, \ldots$ В неравенстве (9.9) коэффициенты $R_{1}^{a}, R_{2}^{a}, Q^{a}$ образуют рекуррентную последовательность $(9.8)$, константа $c_{s}^{(0)}<4$ определена в $(8.16)$ и спектральный радиус $\varrho(\mathbf{A})<3 / 4$.

9.2. Кубические корни. На примере кубических корней $\sqrt[3]{2}, \sqrt[3]{4}$ покажем, как, используя предыдущий метод, можно неравенства (8.16) перенести на другие числа кубического поля $\mathbb{Q}(\sqrt[3]{2})$, отличные от единиц.

Сначала заменим корни $\sqrt[3]{2}, \sqrt[3]{4}$ точкой $\alpha=\left(\alpha_{1}, \alpha_{2}\right)$ с координатами $\alpha_{1}=\sqrt[3]{4}-1, \alpha_{2}=$ $\sqrt[3]{2}-1$. Выбранная точка $\alpha$ уже будет принадлежать треугольной области $\triangle^{\text {int }}$. В рассматриваемом случае нам непосредственно не дан многочлен $f(x)$ из (9.1). Поэтому перейдем сразу к аналогичной (9.2) матрице

$$
M(a)=\left(\begin{array}{ccc}
-1 & -1 & 1 \\
1 & -2 & 0 \\
0 & 1 & 0
\end{array}\right)
$$

Она является унимодулярной матрицей с определителем $\operatorname{det} M(a)=1$ и обладает свойством $(4.4)$, т.е. $M(a) \widehat{\alpha}=\lambda \widehat{\alpha}$, где $\widehat{\alpha}=\left(\begin{array}{lll}\alpha_{1} & \alpha_{2} & 1\end{array}\right)^{T}$ и собственное значение $\lambda=\alpha_{2}=\sqrt[3]{2}-1$. Теперь нужно проверить, что $M(a)$ будет возвратной матрицей, т.е. что она допускает разложение в произведение симметрии треугольника (3.15), (3.16) и элементарных возвратных матриц (3.14). Действительно, такое разложение существует:

$$
M(a)=\left(\begin{array}{ccc}
-1 & -1 & 1 \\
1 & -2 & 0 \\
0 & 1 & 0
\end{array}\right)=\widehat{r}_{1} \cdot \widehat{\delta}_{2}^{12} \cdot \widehat{\delta}_{2}^{02} \cdot\left(\widehat{\delta}_{1}^{12}\right)^{2}
$$

Оно аналогично разложению $(9.3)$ и отличается от него последним множителем $\left(\widehat{\delta}_{1}^{12}\right)^{2}$. Используя разложение (9.11) и применяя лемму 7.3, находим порядковую матрицу (7.16):

$$
\mathbf{M}=R_{1} \cdot D_{2}^{12} \cdot D_{2}^{02} \cdot\left(D_{1}^{12}\right)^{2}=\left(\begin{array}{ccc}
0 & 2 & 1 \\
1 & 2 & 1 \\
0 & 3 & 1
\end{array}\right)
$$

с характеристическим многочленом $c h_{\mathbf{M}}(x)=x^{3}-b_{2} x^{2}-b_{1} x-b_{0}$ из $(7.20)$, равным

$$
\operatorname{ch}_{\mathbf{M}}(x)=\operatorname{det}\left(\begin{array}{ccc}
x & -2 & -1 \\
-1 & x-2 & -1 \\
0 & -3 & x-1
\end{array}\right)=x^{3}-3 x^{2}-3 x-1 .
$$

Поэтому коэффициенты у многочлена $c h_{\mathbf{M}}(x)$ соответственно равны $b_{2}=3, b_{1}=3, b_{0}=1$. 
Снова ради простоты фиксируем параметр $b=0$ и применим теорему 8.1 для нахождения рекуррентных последовательностей $Q^{a}, R_{1}^{a}, R_{2}^{a}$, которые в силу (9.13) имеют вид

$$
Q^{a+3}=3 Q^{a+2}+3 Q^{a+1}+Q^{a}, \quad R_{k}^{a+3}=3 R_{k}^{a+2}+3 R_{k}^{a+1}+R_{k}^{a}
$$

для $k=1,2$. Чтобы определить начальные условия для последовательностей $Q^{a}, R_{1}^{a}, R_{2}^{a}$, нам потребуется знать квадрат порядковой матрицы

$$
\mathbf{M}^{2}=\left(\begin{array}{lll}
2 & 7 & 3 \\
2 & 9 & 4 \\
3 & 9 & 4
\end{array}\right)
$$

Используя (9.12) и (9.15), по формулам (8.17) находим для последовательностей $Q^{a}, R_{1}^{a}, R_{2}^{a}$ начальные условия:

$$
\begin{array}{lll}
Q^{0}=3, & Q^{1}=\sum_{k, l} m_{k l}=11, & Q^{2}=\sum_{k, l} m_{k l}^{2}=43, \\
R_{1}^{0}=1, & R_{1}^{1}=\sum_{k} m_{k 2}=7, & R_{1}^{2}=\sum_{k} m_{k 2}^{2}=25, \\
R_{2}^{0}=1, & R_{2}^{1}=\sum_{k} m_{k 3}=3, & R_{2}^{2}=\sum_{k} m_{k 3}^{2}=11 .
\end{array}
$$

Теперь вернемся к интересующим нас корням $\sqrt[3]{2}, \sqrt[3]{4}$. Покажем, что имеют место следующие неравенства:

$$
\left|Q^{a} \sqrt[3]{4}-R_{1}^{\prime a}\right|+\left|Q^{a} \sqrt[3]{2}-R_{2}^{\prime a}\right| \leqslant c_{s}^{(0)} \varrho(\mathbf{A})^{a}
$$

для всех $a=0,1,2, \ldots$ с константой $c_{s}^{(0)}<6$ и $\varrho(\mathbf{A})<0.51$. Здесь коэффициенты $Q^{a}, R_{1}^{\prime a}$, $R_{2}^{\prime a}$ для $a=0,1,2, \ldots$ являются рекуррентными последовательностями с уравнением $(9.14)$ и начальными условиями

$$
\begin{array}{lll}
Q^{0}=3, & Q^{1}=11, & Q^{2}=43, \\
R_{1}^{\prime 0}=4, & R_{1}^{\prime 1}=18, & R_{1}^{\prime 2}=68, \\
R_{2}^{\prime 0}=4, & R_{2}^{\prime 1}=14, & R_{2}^{\prime 2}=54 .
\end{array}
$$

Действительно, по (6.8) убеждаемся, что калибровочная матрица $\mathbf{A}=A^{(p)} \breve{r}_{2}$ снова имеет два комплексно сопряженных собственных значения $\lambda_{1} \neq \lambda_{2}$, и поэтому в теореме 7.1 нужно применить неравенство $(8.16)$ с константой $c_{s}^{(0)}<6$ и $\varrho(\mathbf{A})<0.51$, из которого будет следовать неравенство (9.17). Вспоминая, что по условию $\alpha_{1}=\sqrt[3]{4}-1$ и $\alpha_{2}=\sqrt[3]{2}-1$, можем неравенство (8.16) переписать в виде (9.17) с новыми коэффициентами $Q^{a}, R_{1}^{\prime a}=Q^{a}+R_{1}^{a}$ и $R_{2}^{\prime a}=Q^{a}+R_{2}^{a}$. Ясно, что эти коэффициенты снова образуют рекуррентные последовательности с уравнением (9.14), но уже с начальными условиями (9.18), получающимися из условий (9.16).

Лагариас [18] обнаружил связь наилучших приближений с рекуррентными последовательностями. В нашем случае также появляются рекуррентные последовательности (8.10), (9.6), (9.14) как следствие теоремы 7.1, когда переходим от ядерных $T^{(i)}$-норм (гексагональных норм), например, к ромбической норме $|x|_{s}=\left|x_{1}\right|+\left|x_{2}\right|$, естественно, с потерей свойства быть наилучшим приближением. При указанном переходе исчезает "геометрия" наилучших приближений.

После того как рекуррентные последовательности найдены, по ним можно оценить скорость приближения (8.16). Так как рассматриваемое здесь кубическое поле $\mathbb{Q}(\sqrt[3]{2})$ является комплексным, то его основная единица будет числом Пизо, поэтому из (9.14) и (9.18) вытекает оценка (9.17), но с бо́льшей константой $c_{s}^{(0)}$. Именно такие иррациональности были исследованы в [10]-[12]. Среди описанных в предложениях 4.1 и 4.2 полей $\mathbb{Q}(\theta)$ также содержатся вещественные кубические поля. Для них непосредственный переход от рекуррентных 
последовательностей к неравенствам (8.16) уже не будет столь очевидным. Переход же от теоремы 7.1 к неравенствам (8.16) продолжает работать и для вещественных полей.

Те же аргументы, что и выше, позволяют заменить оценку (9.17) неравенством

$$
\left|\sqrt[3]{4}-\frac{R_{1}^{\prime a}}{Q^{a}}\right|+\left|\sqrt[3]{2}-\frac{R_{2}^{\prime a}}{Q^{a}}\right| \leqslant \frac{c}{\left(Q^{a}\right)^{3 / 2}}
$$

для всех $a=0,1,2, \ldots$ с константой $c>0$, не зависящей от $a$. Если использовать, скажем, для числа $\sqrt[3]{2}$, разложение в обычную цепную дробь, то числители и знаменатели подходящих дробей будут вычисляться по рекуррентному соотношению второго порядка с переменными коэффициентами. Поскольку для кубических иррациональностей полные разложения в цепные дроби не известны, то на этом пути удается находить лишь конечный набор неравенств. Обрезая же двумерную аппроксимацию (9.19), можно получить одномерную аппроксимацию, например, для кубического корня $\sqrt[3]{2}$ :

$$
\left|\sqrt[3]{2}-\frac{R_{2}^{\prime a}}{Q^{a}}\right| \leqslant \frac{c}{\left(Q^{a}\right)^{3 / 2}} .
$$

В нашем приближении (9.19) используется рекуррентное соотношение (9.14) более высокого, третьего, порядка, но уже с постоянными коэффициентами, что позволяет получать бесконечные сходящиеся последовательности приближений (9.20). Другим методом разложение в цепную дробь для $\sqrt[3]{2}$ было получено в [19] без оценки скорости приближения. Кроме того, интересно сравнить нашу оценку (9.20) с неравенством Бейкера [20]

$$
\left|\sqrt[3]{2}-\frac{P}{Q}\right|>\frac{10^{-6}}{Q^{3}},
$$

имеющим место для всех целых чисел $P$ и $Q$.

\section{Список литературы}

[1] В. Г. Журавлев, “Дифференцирование индуцированных разбиений тора и многомерные приближения алгебраических чисел”, Аналитическая теория чисел и теория функиий. 31, Зап. научн. сем. ПОМИ, 445, ПОМИ, СПб., 2016, 33-92.

[2] А. Я. Хинчин, Цепные дроби, Наука, М., 1978.

[3] Z. Coelho, A. Lopes, L. F. Da Rocha, "Absolutely continuous invariant measures for a class of affine interval exchange maps", Proc. Amer. Math. Soc., 123:11 (1995), 3533-3542.

[4] V. G. Zhuravlev, A. V. Shutov,, Derivaties of Circle Rotations and Similarity of Orbits, Max Planck Institut für Mathematik, Preprint Ser. 62, 2004, 11 pp.

[5] M. Furukado, Sh. Ito, A. Saito, J. Tamura, Sh. Yasutomi, "A new multidimensional slow continued fraction algorithm and stepped surface", Exp. Math., 23:4 (2014), 390-410.

[6] M. Abrate, S. Barbero, U. Cerruti, N. Murru, "Periodic representations for cubic irrationalities", Fibonacci Quart., 50:3 (2012), 252-264.

[7] N. Murru, "On the periodic writing of cubic irrationals and a generalization of Rédei functions", Int. J. Number Theory, 11:3 (2015), 779-799.

[8] Sh. Ito, J. Fujii, H. Higashino, Sh. Yasutomi, "On simultaneous approximation to $\left(\alpha, \alpha^{2}\right)$ with $\alpha^{3}+$ $k \alpha-1=0 "$, J. Number Theory, 99:2 (2003), 255-283.

[9] Q. Wang, K. Wang, Z. Dai, "On optimal simultaneous rational approximation to $\left(\omega, \omega^{2}\right)^{\tau}$ with $\omega$ being some kind of cubic algebraic function", J. Approx. Theory, 148:2 (2007), 194-210.

[10] P. Hubert, A. Messaoudi, "Best simultaneous Diophantine approximations of Pisot numbers and Rauzy fractals", Acta Arith., 124:1 (2006), 1-15. 
[11] N. Chevallier, "Best simultaneous Diophantine approximations of some cubic numbers", J. Théor. Nombres Bordeaux, 14:2 (2002), 403-414.

[12] N. Chevallier, "Best simultaneous Diophantine approximations and multidimensional continued fraction expansions", Mosc. J. Comb. Number Theory, 3:1 (2013), 3-56.

[13] В. Г. ЖКуравлев, “Делящиеся разбиения тора и множества ограниченного остатка", Аналитическая теория чисел и теория функиий. 30, Зап. научн. сем. ПОМИ, 440, ПОМИ, СПб., 2015, 99-122.

[14] В. Г. Журавлев, "Разбиения Рози и множества ограниченного остатка на торе", Труды по теории чисел, Зап. научн. сем. ПОМИ, 322, ПОМИ, СПб., 2005, 83-106.

[15] В. Г. Журавлев, “Двумерные приближения методом делящихся торических разбиений”, Аналитическая теория чисел и теория функиий. 30, Зап. научн. сем. ПОМИ, 440, ПОМИ, СПб., 2015, $81-98$.

[16] В. Г. Журавлев, “Двумерные приближения кубических иррациональностей”, 2016 (в печати).

[17] G. Rauzy, "Nombres algébriques et substitutions", Bull. Soc. Math. France, 110:2 (1982), 147-178.

[18] J. Lagarias, "Best simultaneous Diophantine approximations. I. Groth rates of best approximation denomimators", Trans. Amer. Math. Soc., 272:2 (1982), 545-554.

[19] P. Liardet, P. Stambul, "Algebraic computations with continued fractions", J. Number Theory, 73:1 (1998), 92-121.

[20] A. Baker, "Rational approximations to $\sqrt[3]{2}$ and other algebraic numbers", Quart. J. Math. Oxford Ser. (2), 15 (1964), 375-383. 